\title{
ESTIMACIÓN DEL PELIGRO SÍSMICO DEBIDO A SISMOS INTERPLACA E INSLAB Y SUS IMPLICACIONES EN EL DISEÑO SÍSMICO
}

\author{
Adrián David García Soto ${ }^{(1)}$, Adrián Pozos-Estrada ${ }^{(1)}$, Hanping Hong ${ }^{(2)}$, y Roberto Gómez Martínez ${ }^{(1)}$
}

\section{RESUMEN}

La ocurrencia de grandes sismos causa excitaciones muy intensas del terreno que pueden provocar el colapso de edificios y puentes. Para tomar en cuenta la incertidumbre en esta ocurrencia y en las solicitaciones sísmicas de diseño, la evaluación probabilística del peligro sísmico es empleada para desarrollar espectros de peligro uniforme (EPU) y mapas de contorno de peligro sísmico. Aunque existe mucha información en relación al cálculo del peligro sísmico en México y su inclusión en reglamentos, no hay en la literatura un reporte detallado, que incluya un modelo de peligro sísmico útil para obtener los EPU, para desarrollar mapas de contorno, y para determinar respuestas inelásticas. Además, ya que la demanda de ductilidad de desplazamiento puede diferir para sismos interplaca e inslab, esta diferencia debería incorporarse en la estimación de la confiabilidad estructural. En este estudio se integra un modelo de peligro sísmico útil para estimar los EPU y para desarrollar mapas de contorno para una parte de México; se calcula la contribución al peligro sísmico de cada tipo de sismo; y se estima el nivel de carga sísmica requerido para diseño, considerando las diferencias en la demanda de ductilidad causada por sismos interplaca e inslab.

Palabras Clave: peligro sísmico; mapas de contorno; respuesta inelástica; confiabilidad; diseño sísmico

\begin{abstract}
Large earthquakes cause intensive ground excitations and possibly results in collapse of buildings and bridges. To take into account the uncertainty in earthquake occurrence and seismic load effect in design codes, the probabilistic seismic hazard assessment approach is employed to develop the uniform hazard spectra (UHS) and seismic hazard contour maps. Although there is extensive information concerning Mexican seismic hazard assessment and code making, a detailed report on a hazard model that can be used to evaluate the UHS, to develop the seismic hazard contour maps and to assess inelastic responses is lacking. Furthermore, since the displacement ductility demand could differ for interplate and inslab earthquakes, this difference should be incorporated in estimating the reliability of structures. In the present study a workable seismic hazard model that can be use to estimate UHS and to develop seismic hazard contour maps for part of Mexico is assembled; the contribution of each individual earthquake type to the overall seismic hazard is assessed; and the seismic design load level that is required considering the differences in the ductility demand caused by interplate and inslab earthquakes is estimated.
\end{abstract}

Key Words: seismic hazard; contour maps; inelastic response; reliability; seismic design

Artículo recibido el 22 de abril de 2010 y aprobado para su publicación el 2 de septiembre de 2011.

(1) Instituto de Ingeniería de la UNAM, Ciudad Universitaria, CP 04510, México DF, AGarciaSo@iingen.unam.mx, APozosE@iingen.unam.mx, RGomezM@iingen.unam.mx

(2) Departamento de Ingeniería Civil y Ambiental, The University of Western Ontario, London, Ontario, Canadá, N6A, 5B9, hongh@eng.uwo.ca 


\section{INTRODUCCIÓN}

La ocurrencia de grandes sismos, que son poco frecuentes y aleatorios, causa excitaciones muy intensas del terreno que pueden provocar el colapso de edificios y puentes. La medición del movimiento del terreno está usualmente basada en la aceleración máxima del terreno o en la seudoaceleración (SA). Para tomar en cuenta la incertidumbre en la ocurrencia de un sismo y en la medición del movimiento del terreno, se emplea la evaluación probabilística del peligro sísmico (EPPS) (Cornell 1968, Esteva 1968, McGuire 2004). La EPPS incorpora información de las zonas sísmicas, de los modelos de ocurrencia, de las relaciones magnitud-recurrencia, y de las ecuaciones de predicción del movimiento del terreno (o leyes de atenuación) para estimar el peligro sísmico (e.g., espectros sísmicos de respuesta); también considera la incertidumbre del modelo a través de la actualización Bayesiana. La EPPS es usada para desarrollar espectros de peligro uniforme (EPU) y mapas de contorno de peligro sísmico. Ejemplos de éstos incluyen el cálculo del peligro sísmico en Estados Unidos (Frankel 1995, Frankel et al. 1996) y en Canadá (Adams and Halchuk 2003), donde los modelos de peligro sísmico adoptados (zonas sísmicas, modelos de ocurrencia, relaciones magnitud-recurrencia y leyes de atenuación) se describen detalladamente. Aunque existe una vasta cantidad de publicaciones y actividades de investigación en relación al cálculo del peligro sísmico en México y su inclusión en reglamentos de diseño (Rosenblueth y Gómez 1991, Zúñiga et al. 1997, Ordaz y Reyes 1999, Reyes et al. 2002, Esteva et al. 2002, García 2006, Ordaz et al. 2007, Bojórquez et al. 2008, y Tena-Colunga et al. 2008), no está disponible en la literatura un reporte público y detallado, que contenga la información de un modelo de peligro sísmico en México que pueda ser empleado directamente para obtener los EPU, para desarrollar mapas de contorno de peligro sísmico, y para determinar respuestas inelásticas. Los códigos de diseño de edificios y puentes tienen la intención de alcanzar niveles de confiabilidad consistentes en dichas estructuras o la eficiencia económica de éstas, por lo menos este es actualmente el caso para las mismas categorías estructurales. Con el desarrollo de un código para México, Rosenblueth y Esteva (1972) fueron los pioneros en sentar las bases probabilísticas para seleccionar el nivel de carga sísmica y para la elaboración de reglamentos de diseño. Si bien se dispone de nuevos métodos para evaluar la confiabilidad estructural, así como de nuevas teorías que pueden utilizarse para la toma de decisiones bajo incertidumbre, las bases comúnmente aceptadas y reconocidas para el desarrollo de reglamentos, son las que se sustentan en alcanzar una confiabilidad consistente, y en la teoría del mínimo costo esperado para el ciclo de vida útil (Ellingwood et al. 1980, Madsen et al. 1986, Rackwitz 2000, Ellingwood 2001, Goda y Hong 2006). No obstante, el nivel de confiabilidad implícito para el diseño contra excitaciones sísmicas mediante el uso de un reglamento, no está siempre dilucidado, aún a nivel nocional. Un ejemplo es el actual código de diseño de puentes (SCT 2001), que provee mapas de contorno de la aceleración máxima del terreno y la forma del espectro de diseño, los cuales provienen de los estipulados en el Manual de Obras Civiles (MOC 1993).

La suposición de que los edificios o puentes pueden ser modelados como sistemas de un solo grado de libertad, sirve de base para establecer la fuerza sísmica de diseño en los reglamentos, ya que el nivel de carga sísmica está basado en la SA. En algunos códigos actuales, la fuerza sísmica está definida por el EPU, mientras que en otros se define como una proporción de un espectro de diseño basado en formas paramétricas predeterminadas, que son función de la aceleración máxima del terreno y otros parámetros. El reglamento canadiense para edificios (NBCC 2005) es un ejemplo donde la primera alternativa mencionada anteriormente es adoptada, mientras que la segunda alternativa es considerada en el código canadiense de diseño de puentes (CSA-S6-06 2006), así como en el código AASHTO LRFD (AASHTO 2007), y en el Manual de Obras Civiles de México (MOC 2008). Debido a que sitios dentro de una región grande podrían ser impactados por diferentes tipos de sismo con características de excitación distintas, el 
uso de un espectro de diseño basado en una forma paramétrica predeterminada, podría no garantizar un nivel de confiabilidad consistente o eficiencia económica. Aunado a esto, la demanda de ductilidad de desplazamiento puede diferir para diferentes tipos de sismo, como es el caso de sismos interplaca e inslab (Hong et al. 2010), y esta diferencia debería incorporarse en la estimación de la confiabilidad estructural.

Cabe señalar que los sismos aquí indicados como interplaca, corresponden a los sismos de subducción, es decir, a los sismos que presentan mecanismos de falla inversa debido a la liberación de energía acumulada entre las placas tectónicas a lo largo de los límites convergentes de las mismas. El otro tipo de sismos, aquí referidos como sismos inslab, corresponden a los sismos de falla normal, de profundidad intermedia, que se localizan dentro de las placas oceánicas que subducen bajo el continente, y que ocurren cuando la roca se rompe por las grandes deformaciones que producen los esfuerzos de tensión aplicados sobre el plano de la roca.

Los principales objetivos de este estudio son: integrar un modelo de peligro sísmico que facilite la estimación de los EPU y el desarrollo de mapas de contorno de peligro sísmico, para una parte de México con posibilidades de ser significativamente afectada por sismos interplaca e inslab; calcular la contribución al peligro sísmico de cada uno de estos tipos de sismos en diferentes sitios; estimar el nivel de carga sísmica requerido para diseño, considerando las diferencias en la demanda de ductilidad de desplazamiento causada por los tipos de sismos en cuestión. Para los análisis, no son considerados sismos que no correspondan a los de interplaca e inslab, tales como los sismos "crustal" (Zuñiga et al. 1997, Ordaz et al. 2007). Tampoco se ha incorporado la contribución de la actividad sísmica en los Estados Unidos que afecta el peligro sísmico de México. Este estudio se enfoca sólo en los efectos de los sismos interplaca e inslab en sitios de suelo firme (roca). Otros tipos de sismos como los de corteza, intraplaca o "crustal", que pudieran regir el diseño en periodos estructurales cortos, están fuera del alcance de este estudio.

Para alcanzar los objetivos referidos, el cuerpo de este trabajo se conforma de dos partes principales. Por una parte se aborda el cálculo del peligro sísmico describiendo las zonas sismogenéticas, las relaciones de magnitud-recurrencia, y las leyes de atenuación empleadas para tal efecto. Con la información descrita, es posible emplear el procedimiento de la EPPS desarrollada por Cornell (1968) y Esteva (1968), y se indica en esta parte que en lugar de utilizar integración numérica, se adoptó un procedimiento basado en simulación, el cual también se describe. Lo anterior conduce a la obtención de mapas de contorno de peligro sísmico, así como espectros de peligro uniforme considerando los dos tipos de sismos empleados en este trabajo. La segunda parte principal de la que consta el cuerpo del presente artículo, hace uso de los resultados obtenidos en la primera parte, para abordar la respuesta inelástica de estructuras modeladas como un sistema de un grado de libertad y el cálculo de su nivel de confiabilidad, así como su implicación desde el punto de vista reglamentario. En esta segunda parte se utiliza información desarrolladla en trabajos previos, que al complementarse con lo desarrollado en este trabajo, culmina en la consecución de los objetivos planteados.

\section{ANÁLISIS DEL PELIGRO SÍSMICO}

\section{Metodología y modelo de peligro sísmico}

El cálculo probabilístico del peligro sísmico integra la modelación de la ocurrencia de un sismo, las zonas sismogenéticas, las relaciones de magnitud-recurrencia, y las leyes de atenuación (o ecuaciones de predicción del movimiento del terreno) (Cornell 1968, Esteva 1968, McGuire 2004). 
El proceso de ocurrencia de un sismo es comúnmente modelado como Poisoniano, aunque también se ha considerado como un proceso no Poisoniano. En el primer caso, el tiempo de llegada entre dos temblores sucesivos se asume como una variable con distribución exponencial con un parámetro determinado por la tasa de ocurrencia sísmica, mientras que para el segundo, dicho tiempo entre eventos es usualmente considerado una variable del tipo lognormal, gama, o Gaussiana inversa (Rosenblueth y Jara 1991). Más aún, se asume frecuentemente que la ocurrencia de un sismo es estadísticamente homogénea en magnitud y en ubicación dentro de una zona sísmica. Para definir las características del peligro sísmico en una parte de México, consideramos que son adecuadas las zonas sísmicas proporcionadas por Ordaz y Reyes (1999) cerca de la Costa del Pacífico Mexicano. Estas zonas se separan en dos grupos como se aprecia en las figuras $1 \mathrm{a}$ y $1 \mathrm{~b}$, y cuyos parámetros se enlistan en la tabla 1. El primer grupo corresponde a sismos pequeños y moderados con magnitudes menores a 7 , y el segundo grupo a sismos con magnitudes mayores a 7. Cada zona sísmica está definida por un polígono; sus contornos fueron digitalizados a partir de las figuras graficadas por Ordaz y Reyes (1999). La ocurrencia de sismos interplaca (i.e., eventos en la frontera de la placa) se modela dentro de estas zonas sísmicas. Se considera que los sismos inslab, que ocurren dentro de la placa oceánica subducida debajo de la placa continental, tienen origen en las grandes zonas sísmicas mostradas en la figura 1c y en la tabla 1 (Jaimes y Reinoso 2006).

Para las zonas sísmicas mostradas en la figura 1a con $M_{W}<7$, la relación magnitud-recurrencia, $\lambda\left(M_{W}\right)$, está definida por,

$\lambda\left(M_{W}\right)=\lambda_{0} \frac{\exp \left(-\beta M_{W}\right)-\exp \left(-\beta M_{U}\right)}{\exp \left(-\beta M_{L}\right)-\exp \left(-\beta M_{U}\right)}$

y para las zonas sísmicas mostradas en la figura $1 \mathrm{~b} \operatorname{con} M_{W}>7$, por,

$$
\lambda\left(M_{W}\right)=\lambda(7)\left(1-\Phi\left(\left(M_{W}-m_{M}\right) / s_{M}\right)\right)
$$

donde $M_{\mathrm{W}}$ representa el momento de magnitud, $M_{\mathrm{L}}=4.5, M_{\mathrm{U}}=7, m_{\mathrm{M}}=7.5, s_{M}=0.3, \Phi()$ denota la función de distribución de probabilidad normal estándar, y $\lambda_{0}, \beta$, y $\lambda(7)$ son parámetros de modelación. Los valores de los parámetros incluyendo $\lambda_{0}, \beta$, y $\lambda(7)$ son tomados directamente del estudio de Ordaz y Reyes (1999) y listados en la tabla $1 ; \lambda(7)$, en lugar de $\lambda_{0}$, se utiliza como la tasa de ocurrencia sísmica para sismos con magnitud mayor a 7.0. Para los sismos inslab dentro de las zonas sísmicas mostradas en la figura 1c, se considera que la ec. 1 también se puede emplear, pero con $M_{\mathrm{L}}=4.5$, y $M_{\mathrm{U}}=7.9$ para la región denominada Profundidad Intermedia Oeste, y $M_{\mathrm{U}}=7.88$ para la región denominada Profundidad Intermedia Centro (Jaimes y Reinoso 2006).

La ec. 1 implica que la función de distribución de probabilidad de la magnitud $M_{\mathrm{w}}, F_{\mathrm{M}}(m)$, se puede expresar como,

$$
F_{M_{W}}\left(M_{w}\right)=\frac{1-\exp \left(-\beta\left(M_{W}-M_{L}\right)\right)}{1-\exp \left(-\beta\left(M_{U}-M_{L}\right)\right)}
$$

mientras que la función de distribución de probabilidad asociada con la ec. 2 está dada por, 
a)

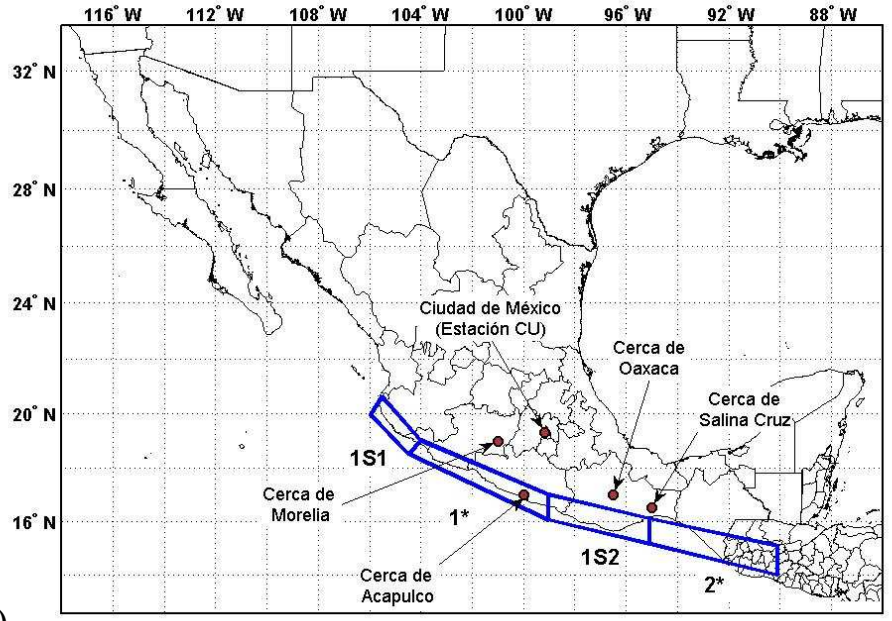

b)
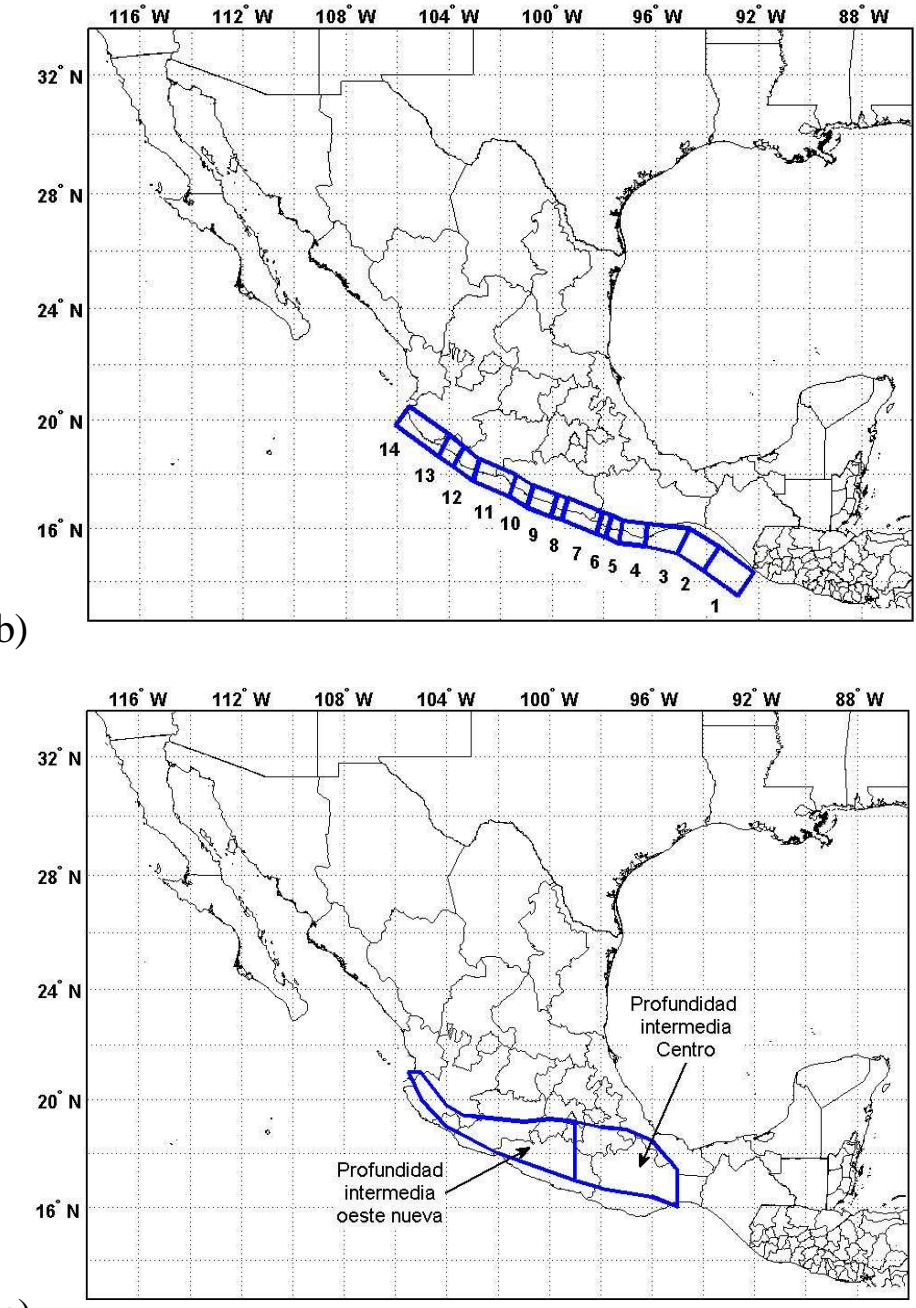

c)

Figura 1. Zonas sísmicas de acuerdo a Ordaz y Reyes (1999) y Jaimes y Reinoso (2006) 


$$
F_{M_{W}}\left(M_{w}\right)=\frac{\Phi\left(\left(M_{W}-m_{M}\right) / s_{M}\right)-\Phi\left(\left(7-m_{M}\right) / s_{M}\right)}{\left(1-\Phi\left(\left(7-m_{M}\right) / s_{M}\right)\right)}
$$

De las ecs. 1 y 2 también se infiere que el tiempo de llegada de un sismo está distribuido exponencialmente, su media es igual a $1 / \lambda_{0}$ y $1 / \lambda(7)$ para las zonas símicas correspondientes, siempre que la ocurrencia esté representada por un proceso Poisoniano. En el presente estudio se adopta el modelo de ocurrencia Poisoniano. Esta suposición ha sido ampliamente adoptada en la literatura (ver las referencias enlistadas en Pozos-Estrada et al. 2008). Lo anterior conlleva a la utilización de caracterizaciones simples del peligro sísmico, independientes del tiempo.

Si bien existe un estudio sobre la traza de la interface de las placas continental y subducida (Pardo y Suárez 1995), debido a que existe incertidumbre acerca de la profundidad de los sismos, y por lo tanto acerca de la superficie que define la profundidad de los mismos, en este estudio se consideraron profundidades deterministas de $10.45 \mathrm{~km}$ para sismos interplaca, y de $64.56 \mathrm{~km}$ para sismos inslab, las que representan, respectivamente, la profundidad promedio de los sismos asociados con los registros utilizados en el desarrollo de las leyes de atenuación descritas en las líneas subsecuentes. Las formas funcionales adoptadas para las relaciones de atenuación son,

$$
\log _{10}(Y)=c_{1}+c_{2}\left(M_{W}-6\right)+c_{3}\left(M_{W}-6\right)^{2}+c_{4} \log _{10} R+c_{5} R+\varepsilon
$$

para la estación de CU (localizada en Ciudad Universitaria), desarrollada para sismos interplaca (Reyes et al. 2002),

$\log _{10} Y=c_{1}+c_{2} M_{w}+c_{3} R-c_{4} \log _{10}\left(R+c_{5} 10^{c_{6} M_{w}}\right)+c_{7} H+\varepsilon$,

para sismos interplaca (García 2006), y

$$
\log _{10} Y=c_{1}+c_{2} M_{w}+c_{3} R-c_{4} \log _{10} R+c_{5} H+\varepsilon,
$$

para sismos inslab (García et al. 2005). Se considera que la ec. 5 es aplicable únicamente para la ciudad de México, mientras que las ecs. 6 y 7 son aplicables a México central. Datos sobre las distancias consideradas para el desarrollo de las leyes de atenuación son proporcionados más adelante; el lector puede revisar las referencias García (2006) y García et al. (2005) para mayor detalle sobre la aplicabilidad de las leyes de atenuación que corresponden a las ecs. 6 y 7. En las ecuaciones 5 a 7, $Y$ denota la aceleración máxima del terreno o la SA, $S_{A}\left(T_{\mathrm{n}}, \xi\right)$, como una fracción de la aceleración de la gravedad $(\mathrm{g})$; $T_{\mathrm{n}}$ (s) es el periodo fundamental de vibración; $\xi$ es el porcentaje de amortiguamiento crítico, el cual es igual a 5\% a lo largo de este estudio; $M_{W}$ es el momento de magnitud de un sismo; el término $\varepsilon$ es el error considerado como una variable normal con media cero y desviación estándar $\sigma=\left(\sigma_{\mathrm{r}}^{2}+\sigma_{\mathrm{e}}{ }^{2}+\sigma_{\mathrm{c}}{ }^{2}\right)^{0.5}$, donde $\sigma_{\mathrm{r}}$ y $\sigma_{\mathrm{e}}$ denotan las desviaciones estándar debido a las variabilidades intra-evento e inter-evento, respectivamente, y $\sigma_{\mathrm{c}}$ es la desviación estándar que toma en consideración la variabilidad debido a la orientación aleatoria (Boore et al., 1997).

En la ec. 5, $R(\mathrm{~km})$ es la distancia más cercana a la superficie de falla; y $c_{\mathrm{i}}, i=1, \ldots, 5$, representa coeficientes de regresión. En la ec. $6, R(\mathrm{~km})$ es la distancia más cercana a la superficie de falla para eventos con $M_{w}>6.0$, o la distancia hipocentral para el resto; $H(\mathrm{~km})$ es la profundidad focal; $c_{4}=1.82$ $0.16 M_{w} ; \mathrm{y} c_{1}, c_{2}, c_{3}, \mathrm{y} c_{7}$ son coeficientes de regresión. En la ec. 7, $c_{\mathrm{i}}, i=1,2,3,4,5$, también representa coeficientes de regresión; $c_{4}=1 ; R=\left(R_{\text {cld }}{ }^{2}+\Delta_{0}\right)^{1 / 2} ; R_{\text {cld }}(\mathrm{km})$ es la distancia más cercana a la superficie de 
falla para eventos con $M_{w}>6.5$, o la distancia hipocentral para el resto; $H(\mathrm{~km})$ es la profundidad focal; y $\Delta_{0}=0.0075 \times 10^{0.507 \mathrm{M}_{w}}$ es un término de saturación de la fuente definido por Atkinson y Boore (2003).

Tabla 1. Parámetros de tasas de excedencia para las zonas sísmicas mostradas en la figura 1(obtenidos en Ordaz y Reyes 1999, y Jaimes y Reinoso 2006)

\begin{tabular}{|c|c|c|c|}
\hline Zona & $\begin{array}{l}\lambda_{0} \text { ó } \lambda(7) \\
(1 / \mathrm{año})\end{array}$ & $\beta$ & $\begin{array}{l}\text { Puntos (latitud, longitud) empelada para definir los límites de } \\
\text { la zona sísmica }\end{array}$ \\
\hline 1S1 & 2.014 & 1.827 & $\begin{array}{c}19.975,-105.975 ; 18.513,-104.475 ; 19.025,-104 ; 20.65,- \\
105.525\end{array}$ \\
\hline $1 *$ & 4.792 & 1.547 & $\begin{array}{c}18.513,-104.475 ; 16.063,-99.030 ; 17.025,-99.030 ; 19.025,- \\
104\end{array}$ \\
\hline $1 \mathrm{~S} 2$ & 6.717 & 1.847 & $\begin{array}{c}16.063,-99.030 ; 15.150,-95.070 ; 16.120,-95.070 ; 17.0250,- \\
99.030\end{array}$ \\
\hline $2 *$ & 18.938 & 2.059 & $\begin{array}{c}15.150,-95.070 ; 13.975,-90.075 ; 15.100,-90.075 ; 16.120,- \\
95.070\end{array}$ \\
\hline 1. Chiapas & 0.0369 & & $\begin{array}{c}14.390,-94.145 ; 13.451,-92.804 ; 14.370,-92.168 ; 15.318,- \\
93.510\end{array}$ \\
\hline 2. Brecha de & & & $15.045,-95.164 ; 14.390,-94.145 ; 15.318,-93.510 ; 15.994,-$ \\
\hline Tehuantepec & 0.03344 & & 94.660 \\
\hline 3. Oaxaca Este & 0.02793 & & $\begin{array}{c}15.318,-96.405 ; 15.045,-95.164 ; 15.994,-94.660 ; 16.185,- \\
96.314 \\
15.440-97430 \cdot 15318-96.405 \cdot 16185-96314 \cdot 16.286 \text { - }\end{array}$ \\
\hline 4. Oaxaca Centro I & 0.01898 & & 97.273 \\
\hline 5. Oaxaca Centro II & 0.01339 & & $\begin{array}{c}15.652,-97.935 ; 15.440,-97.430 ; 16.286,-97.273 ; 16.478,- \\
97.676 \\
15.783,-98.288 ; 15.652,-97.935 ; 16.478,-97.676 ; 16.610,-\end{array}$ \\
\hline 6. Oaxaca Oeste & 0.01116 & & $\begin{array}{l}98.040 \\
16308 \quad 09640 \cdot 15783-98288 \cdot 16610-08040 \cdot 17134-\end{array}$ \\
\hline 7. Ometepec & 0.02899 & & $16.419,-100.083 ; 16.308,-99.640 ; 17.134,-99.392 ; 17.265,-$ \\
\hline 8. San Marcos & 0.01116 & & $\begin{array}{c}99.805 \\
16.762,-101.002 ; 16.419,-100.083 ; 17.265,-99.805 ; 17.598,-\end{array}$ \\
\hline 9. Guerrero & 0.02232 & & 100.733 \\
\hline 10. Petatlán & 0.01563 & & $\begin{array}{c}17.165,-101.667 ; 16.762,-101.002 ; 17.598,-100.733 ; 17.983, \\
-101.385\end{array}$ \\
\hline 11. Michoacán & 0.03356 & & $\begin{array}{c}\text { 17.740, }-103.080 ; 17.165,-101.667 ; 17.983,-101.385 ; 18.558, \\
-102.808\end{array}$ \\
\hline 12. Colima & 0.01786 & & $\begin{array}{c}18.265,-103.847 ; 17.740,-103.080 ; 18.558,-102.808 ; 19.032, \\
-103.423\end{array}$ \\
\hline 13. Brecha de Colima & 0.01675 & & $\begin{array}{c}18.668,-104.432 ; 18.265,-103.847 ; 19.032,-103.423 ; 19.446 \text {, } \\
-103.968\end{array}$ \\
\hline 14. Jalisco & 0.04566 & & $\begin{aligned} & 19.818,-106.086 ; 18.668,-104.432 ; 19.446,-103.968 ; 20.505 \\
&-105.562 \\
& 21,-105.5 ; 20,-105 ; 19,-104 ; 18,-102 ; 17,-99 ; 19.2,-99\end{aligned}$ \\
\hline Prof. Intermedia Oeste & & & $19.3,-100 ; 19.2,-101 ; 19.4,-103 ; 19.4,-103.3 ; 19.8,-104 ; 21$ \\
\hline Nueva (Sismos Inslab) & 2.161 & 1.699 & -105 \\
\hline $\begin{array}{l}\text { Prof. Intermedia Centro } \\
\quad(\text { Sismos Inslab })\end{array}$ & 1.714 & 1.576 & $\begin{array}{c}19.2,-99 ; 17,-99 ; 16.7,-98 ; 16.4,-96 ; 16,-95 ; 17.4,-95 ; 18.5 \\
-96 ; 18.9,-97 ; 19,-98\end{array}$ \\
\hline
\end{tabular}

Los coeficientes, $c_{\mathrm{i}}$, obtenidos por Reyes et al. (2002), García et al. (2005), y García (2006) para las ecs. 5 a 7, están basados en la media cuadrática; nosotros preferimos usar las leyes de atenuación desarrolladas con base en la media geométrica (definida como la raíz cuadrada del producto de las seudoaceleraciones para cada componente), ya que éstas derivan en la desviación estándar en las ecuaciones de predicción de movimiento del terreno para una orientación aleatoria. Los coeficientes que provienen de emplear la media geométrica para las ecs. 5 a 7 , se pueden encontrar en las publicaciones de 
Pozos-Estrada et al. (2008) y Hong et al. (2009) y se muestran en la tabla 2. Estos coeficientes se obtuvieron recurriendo a la misma serie de registros seleccionados por Reyes et al. (2002), García et al. (2005), y García (2006), pero empleando el algoritmo de regresión de Joyner y Boore (1993). Nótese que una corrección de línea base (Boore 2005) fue aplicada a los registros considerados por García et al. (2005) y García (2006); nótese además que menos de 10 registros fueron excluidos, ya que éstos conducían a respuestas poco realistas (Hong et al. 2010). Los valores de $Y$ que se predicen utilizando los coeficientes mostrados en la tabla 2, son comparables con aquellos cuya predicción se obtiene mediante los coeficientes que se basan en la media cuadrática.

Los coeficientes de la ley de atenuación para la estación localizada en Ciudad Universitaria en la Cd. de México (suelo firme) fueron desarrollados para sismos interplaca (subducción) cuyos registros corresponden a momentos de magnitud $\left(M_{\mathrm{w}}\right)$ entre 6.1 y 8.1, y distancias epicentrales comprendidas entre 280 y $466 \mathrm{~km}$ (Reyes et al. 2002). En lo referente a los coeficientes para la ley de atenuación para sismos interplaca (subducción), estos fueron desarrollados para sitios sobre roca firme comprendidos entre la costa y el Cinturón Volcánico Mexicano; el número de eventos sísmicos es 40, con momentos de magnitud $\left(M_{\mathrm{w}}\right)$ entre 5 y 8 , y profundidad focal $(\mathrm{H})$ entre 8 y $29 \mathrm{~km}$, y se consideró una distancia más próxima al plano de ruptura de hasta $400 \mathrm{~km}$ (García 2006). Finalmente, los coeficientes para la ley de atenuación para sismos inslab (falla normal, profundidad intermedia), fueron desarrollados para sitios en México Central sobre roca firme; el número de eventos sísmicos es 16 (registrados a distancias locales y regionales de hasta $400 \mathrm{~km})$, con momentos de magnitud $\left(M_{\mathrm{w}}\right)$ entre 5.2 y 7.4 , y profundidad focal $(H)$ entre 35 y $138 \mathrm{~km}$ (García et al. 2005). Nótese que a diferencia de la ley de atenuación propuesta por Reyes et al. (2002), las relaciones funcionales propuestas por García (2006) y por García et al. (2005), incluyen la profundidad focal $H$, debido a que en esos estudios se encontró dependencia entre la aceleración máxima del terreno (o la seudoaceleración) y la profundidad focal.

Debe enfatizarse que las ecs. 6 y 7 no rigen para sitios dentro del Valle de México ni dentro del Cinturón Volcánico Mexicano, debido a las características particulares de propagación a lo largo del Cinturón Volcánico Mexicano y de respuesta de los sitios dentro del Valle de México (García 2006). La ec. 5 es válida para $M_{W} \leq 8.1$; en caso de eventos con $M_{W}>8.1$, sus magnitudes son consideradas iguales a 8.1 (Reyes, comunicación personal 2007). La misma condición se considera para la ec. 6.

\section{Procedimiento de análisis}

Para este estudio, consideramos que el peligro sísmico puede ser representado por la aceleración máxima del terreno y la SA, y está denotado por $Y$. El procedimiento más popular de la EPPS fue desarrollado por Cornell (1968) y Esteva (1968) (ver McGuire 2004) como se ilustra en la figura 2. Las suposiciones para tal cálculo incluyen que: la ocurrencia de un sismo en cada zona sísmica es uniformemente distribuida; se conocen las leyes de atenuación para predecir la aceleración máxima del terreno y la SA, así como sus modelos probabilísticos asociados (para los términos de error); un sismo puede ser representado como una fuente puntual; y que el modelo probabilista del tiempo de llegada entre sismos para cada zona está asignado. Basados en dichas suposiciones, la función de densidad de probabilidad de $Y, F_{\mathrm{Y}}(y)$, puede expresarse como,

$$
F_{Y}(y)=1-\sum_{\substack{\text { zona } \\ \text { sísmica, }=1}}^{\mathrm{n}_{\mathrm{s}}} \int_{\Omega_{\mathbf{X}_{i}}}\left(\lambda_{M_{\min }, i} \int_{\Omega_{i}}\left(1-F_{Y \mid M_{W}, R, i}(y)\right) f_{M_{W}, R, i}\left(m_{W}, r\right) d m_{W} d r\right) f_{X_{i}}(x) d x
$$

donde $n_{\mathrm{s}}$ denota el número total de zonas sísmicas; $\lambda_{M \text { min,i }}$ es la relación de ocurrencia de los eventos 
sísmicos con $M_{W} \geq M_{\min }$ para la $i$-ésima zona sísmica; $f_{M_{W}, R, i}\left(m_{W}, r\right)$ denota la función de densidad de probabilidad conjunta de $M_{\mathrm{W}}$ y $R ; \Omega_{\mathrm{i}}$ denota el dominio de $M_{\mathrm{W}}$ y $R$ para la $i$-ésima zona sísmica; $F_{Y \mid M_{W}, R, i}(y)$ es la función de densidad de probabilidad conjunta de $Y$ condicionada a $M_{\mathrm{W}}, R$, y la $i$-ésima

Tabla 2a. Coeficientes de atenuación desarrollados con base en la media geométrica considerando el sitio de Ciudad Universitaria para sismos mexicanos de subducción (Pozos-Estrada et al. 2008)

\begin{tabular}{ccccccc}
\hline$T_{n}(\mathrm{~s})$ & $c_{1}$ & $c_{2}$ & $c_{3}$ & $c_{4}$ & $c_{5}$ & $\sigma$ \\
\hline 0.1 & 2.604 & 0.390 & 0.003 & -0.5 & -0.002 & 0.139 \\
0.2 & 2.963 & 0.221 & 0.053 & -0.5 & -0.003 & 0.127 \\
0.3 & 3.080 & 0.218 & 0.058 & -0.5 & -0.003 & 0.137 \\
0.4 & 2.905 & 0.516 & -0.030 & -0.5 & -0.003 & 0.159 \\
0.5 & 3.020 & 0.429 & 0.002 & -0.5 & -0.003 & 0.144 \\
0.7 & 3.002 & 0.435 & 0.013 & -0.5 & -0.003 & 0.146 \\
1 & 2.881 & 0.483 & 0.000 & -0.5 & -0.003 & 0.142 \\
2 & 2.571 & 0.633 & -0.046 & -0.5 & -0.002 & 0.203 \\
3 & 2.321 & 0.789 & -0.115 & -0.5 & -0.002 & 0.195 \\
0 & 2.653 & 0.340 & 0.029 & -0.5 & -0.003 & 0.135 \\
\hline
\end{tabular}

Tabla 2b. Coeficientes de atenuación para la media geométrica considerando los registros de los sismos

\begin{tabular}{cccccccc}
\hline$T_{n}(\mathrm{~s})$ & $c_{1}$ & $c_{2}$ & $c_{3}$ & $c_{5}$ & $c_{6}$ & $c_{7}$ & $\sigma$ \\
\hline 0.10 & 3.040 & 0.091 & -0.0045 & 0.0075 & 0.496 & -0.0020 & 0.39 \\
0.20 & 2.609 & 0.144 & -0.0034 & 0.009 & 0.475 & -0.0041 & 0.36 \\
0.30 & 2.256 & 0.178 & -0.0026 & 0.005 & 0.492 & -0.0058 & 0.36 \\
0.40 & 1.841 & 0.212 & -0.0020 & 0.004 & 0.504 & -0.0036 & 0.37 \\
0.50 & 1.542 & 0.238 & -0.0015 & 0.003 & 0.515 & -0.0030 & 0.36 \\
0.70 & 1.058 & 0.282 & -0.0009 & 0.002 & 0.512 & -0.0029 & 0.36 \\
1.00 & 0.734 & 0.301 & -0.0005 & 0.002 & 0.509 & -0.0050 & 0.36 \\
2.00 & -0.314 & 0.391 & -0.0002 & 0.002 & 0.489 & -0.0052 & 0.33 \\
3.00 & -0.869 & 0.432 & -0.0003 & 0.002 & 0.49 & -0.0049 & 0.35 \\
0 & 2.545 & 0.108 & -0.0037 & 0.0075 & 0.474 & -0.0024 & 0.35 \\
\hline
\end{tabular}

Tabla 2c. Coeficientes de atenuación para la media geométrica (i.e., $A_{\mathrm{GM}}\left(T_{\mathrm{n}}\right)$ ) considerando los registros de los sismos inslab ( $c_{4}$ es igual a 1) (Hong et al. 2009)

\begin{tabular}{cccccc}
\hline$T_{\mathrm{n}}(\mathrm{s})$ & $c_{1}$ & $c_{2}$ & $c_{3}$ & $c_{5}$ & $\sigma$ \\
\hline 0.10 & 0.387 & 0.549 & -0.0040 & 0.0077 & 0.35 \\
0.20 & -0.020 & 0.595 & -0.0036 & 0.0068 & 0.30 \\
0.30 & -0.355 & 0.640 & -0.0032 & 0.0048 & 0.29 \\
0.40 & -0.653 & 0.658 & -0.0027 & 0.0047 & 0.28 \\
0.50 & -0.907 & 0.687 & -0.0024 & 0.0034 & 0.28 \\
0.70 & -1.346 & 0.714 & -0.0019 & 0.0038 & 0.29 \\
1.00 & -1.931 & 0.781 & -0.0016 & 0.0029 & 0.29 \\
2.00 & -2.903 & 0.867 & -0.0012 & 0.0014 & 0.28 \\
3.00 & -3.513 & 0.916 & -0.0008 & 0.0008 & 0.27 \\
0 & -0.109 & 0.569 & -0.0039 & 0.0070 & 0.30 \\
\hline
\end{tabular}

zona sísmica (la cual puede ser derivada de las características de la ley de atenuación aplicable); $\boldsymbol{X}_{\mathrm{i}}$ representa a las variables aleatorias epistémicas (e.g., incertidumbre en los parámetros de modelación que se emplean para definir los modelos de la ley de atenuación y zonas sísmicas), para la $i$-ésima zona 
sísmica cuya función de densidad de probabilidad conjunta está definida por $f_{X i}(\boldsymbol{x})$; y $\Omega_{X \mathrm{i}}$ denota el dominio de $\boldsymbol{X}_{\mathrm{i}}$ para la $i$-ésima zona sísmica. En lugar de utilizar integración numérica como fue hecho por McGuire (1976), la ec. 8 puede ser evaluada mediante técnicas de simulación para modelos de ocurrencia tanto Poisonianos como no Poisonianos.

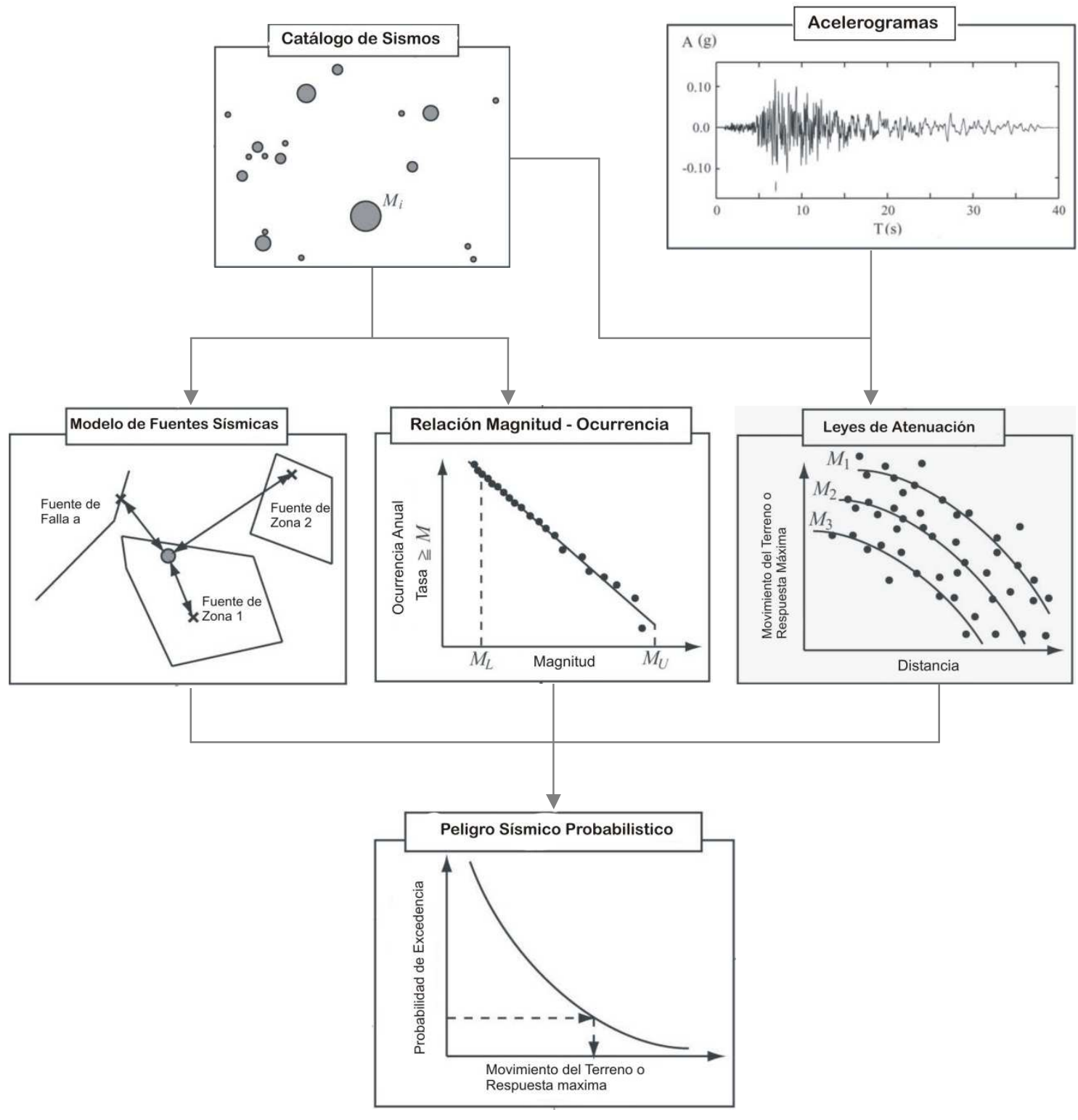

Figura 2. Ilustración del procedimiento para determinar el peligro sísmico (modif. de Hong et al. 2006)

En el presente estudio se adopta un procedimiento basado en simulación, siguiendo los pasos descritos a continuación, para la EPPS y considerando un período de observación igual a $T_{\mathrm{T}}$ (Hong et al. 2006): 
1) Muestrear las variables aleatorias epistémicas en $\boldsymbol{X}_{\mathrm{j}}$ para la $j$-ésima zona símica, $j=1, \cdots, n_{s}$;

2) Muestrear el tiempo de llegada entre sismos $\tau_{\mathrm{j}}$ de acuerdo al modelo de ocurrencia adoptado y fijar $t_{\mathrm{j}}=\tau_{\mathrm{j}}, j=1, \cdots, n_{s}$; encontrar la $i$-ésima zona sísmica cuyo $t_{\mathrm{i}}$ es igual a $\min \left(t_{1}, \cdots, t_{\mathrm{n}}\right)$;

3) Muestrear $M_{\mathrm{W}}$ (ver ecs. 3 y 4 ) y la localización del sismo, así como el término de error de la ley de atenuación pertinente (ver ecs. 5 a 7); medir el movimiento del terreno en términos de $Y, y$; muestrear $\tau_{\mathrm{i}}$ de acuerdo a su modelo de ocurrencia y calcular $t_{\mathrm{i}}=t_{\mathrm{i}}+\tau_{\mathrm{i}}$;

4) Repetir el paso 3$)$ si $\min \left(t_{1}, \cdots, t_{\mathrm{n}}\right)$ es menor que $T_{\mathrm{T}}$.

Al repetir los pasos 1) a 4) $n_{\mathrm{E}}$ ciclos, se obtienen muestras para $n_{\mathrm{E}} \times T_{\mathrm{T}}$ años que se pueden subdividir en varios intervalos de $T_{\mathrm{d}}$ años, los que están igualmente espaciados sin sobreponerse. Los máximos efectos sísmicos que corresponden a cada intervalo de tiempo, pueden utilizarse para formar una secuencia de efectos máximos del movimiento del terreno para un periodo de $T_{\mathrm{d}}$ años. La incertidumbre epistémica no se considera en los análisis numéricos presentados en las secciones subsecuentes, debido a la carencia de diferentes (pero igualmente creíbles) modelos de peligro sísmico.

\section{Peligro sísmico y mapas de contorno}

Para investigar el impacto que los sismos interplaca e inslab tienen en el peligro sísmico estimado, se evalúan tres conjuntos de resultados utilizando el procedimiento descrito anteriormente, y se presentan en forma de mapas de contorno de la aceleración máxima del terreno y la SA en términos de la media, coeficiente de variación (cov), y valores asociados a un período de retorno dado. Los conjuntos de resultados son para sismos interplaca, sismos inslab, y una combinación de ambos. Para el análisis se considera un ciclo de 75 simulaciones, cada uno con 2500 años de observación, traduciéndose estos valores en un intervalo de simulación de 187,500 años de actividad sísmica. Para evaluar los mapas de contorno se usa un sistema de malla rectangular con una distancia de $0.5^{\circ}$ entre dos puntos adyacentes en la malla (a lo largo de la latitud y de la longitud). Se consideran valores de $Y, y_{T}$, asociados a un periodo de retorno $T$ igual a 2475 años (i.e., probabilidad de excedencia de $2 \%$ en 50 años), y se calculan la media y cov de las distribuciones de probabilidad ajustadas en el extremo superior de las muestras.

Los resultados obtenidos se presentan en las figuras 3, 4 y 5 para los casos que consideran únicamente sismos interplaca, únicamente sismos inslab, y ambos tipos de sismos, respectivamente. Para ahorrar espacio, sólo se muestran los mapas de contorno de la aceleración máxima del terreno $\left(T_{n}=0 \mathrm{~s}\right)$ y la SA para $T_{n}=0.5$ y $2 \mathrm{~s}$, aunque también se calcularon los resultados para otros valores de $T_{\mathrm{n}}$ como ayuda para realizar algunas observaciones que se presentan más adelante. Si bien los resultados para el valle de la Ciudad de México y para el Cinturón Volcánico Mexicano son incluidos por complementariedad, éstos no deben usarse, ya que como se mencionó anteriormente las ecs. 6 y 7 no aplican para estos sitios (García 2006).

La figura 3 muestra mapas de contorno de peligro símico en términos de la media $m_{\mathrm{Y}}$, cov $v_{\mathrm{Y}}, \mathrm{y}$ valores de la aceleración máxima del terreno y la SA, $Y$, para un período de retorno igual a 2475 años, $y_{2745}$. Nótese que el mapa con los valores para un periodo de retorno seleccionado, no proporciona una visión completa del peligro sísmico, mientras que los mapas de la media y el coeficiente de variación, podrían emplearse para proporcionar una caracterización probabilística completa del peligro sísmico, siempre que la distribución de probabilidad sea asignada. Esto último es especialmente importante para estimar la confiabilidad de estructuras sometidas a carga sísmica. Como se esperaba, al observar la figura 3, los mapas indican que, $m_{\mathrm{Y}}$, y $y_{2475}$ a lo largo y cerca de la costa del Pacífico Mexicano son los más altos para sismos interplaca 


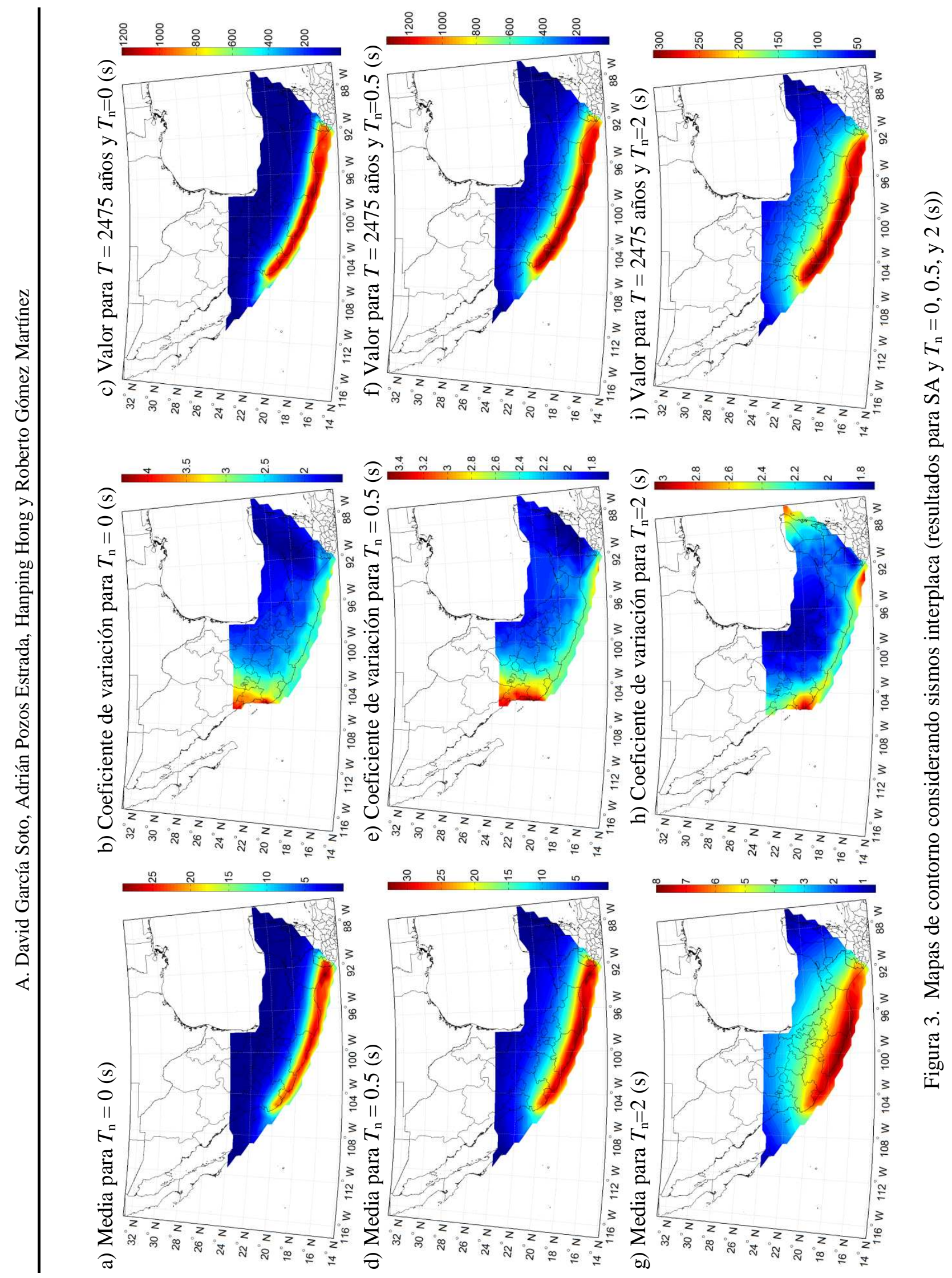




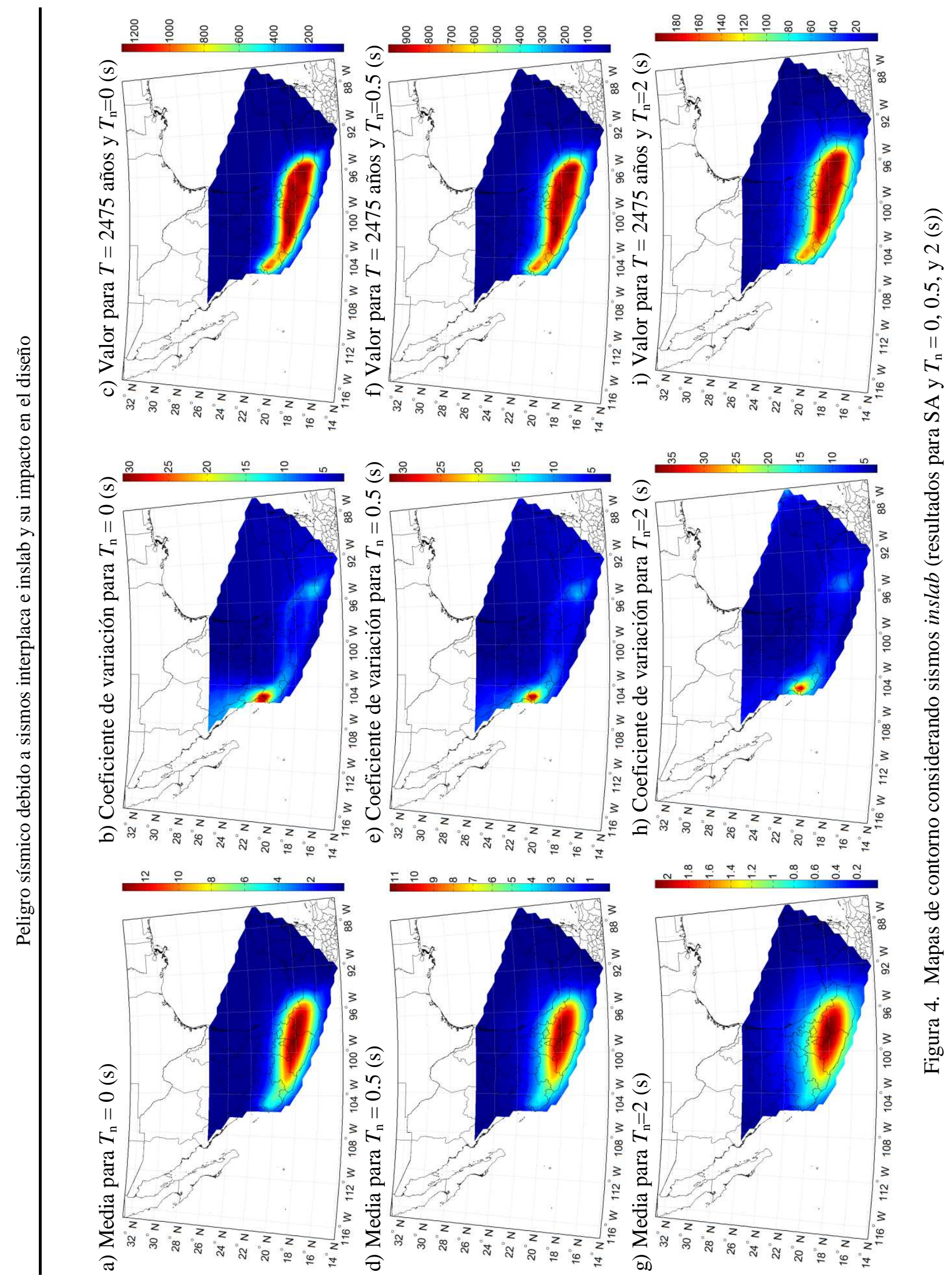

ले 


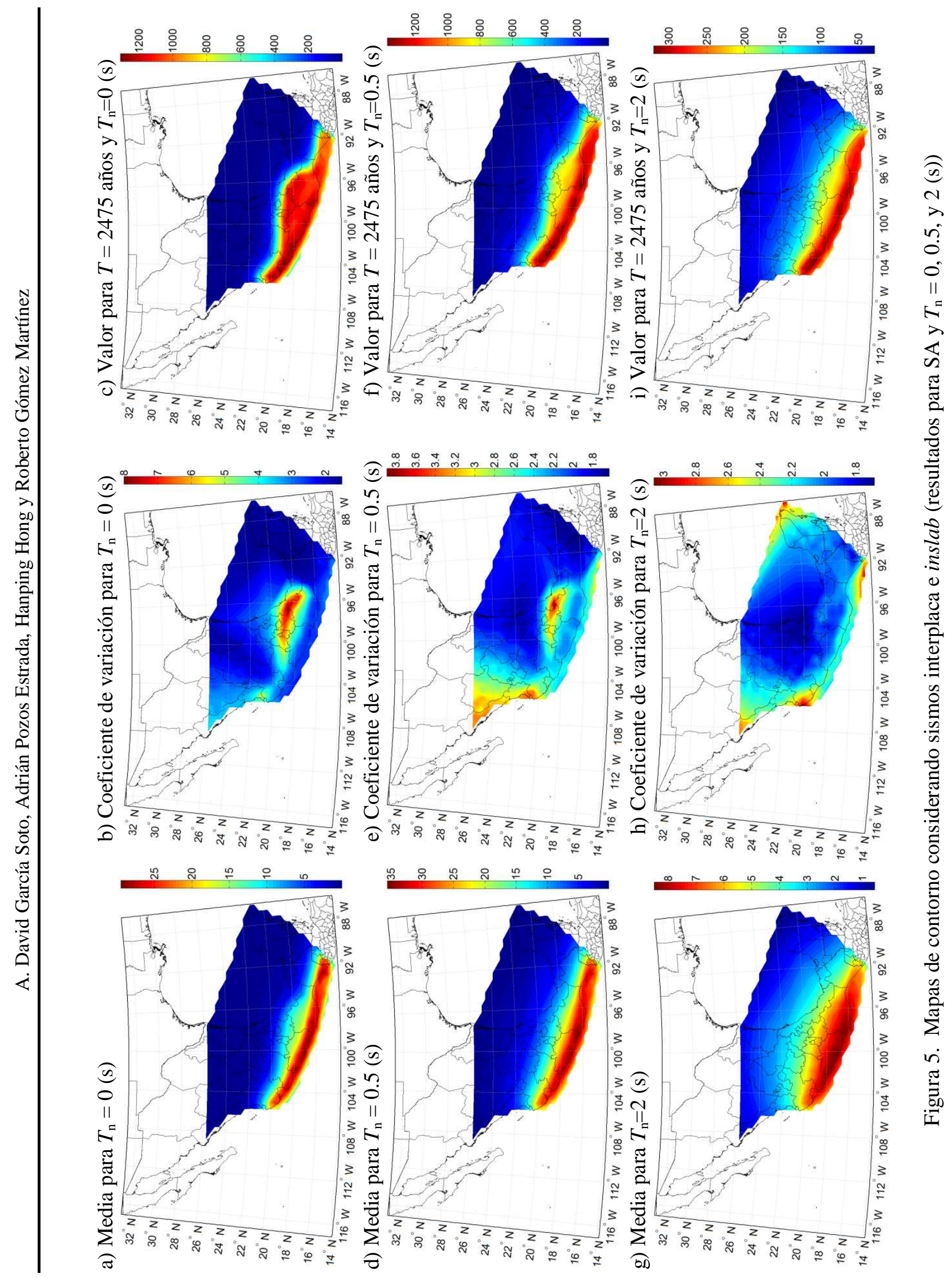


Los valores de $v_{\mathrm{Y}}$ varían aproximadamente entre 2 y 3 para la mayoría de los sitios; estos coeficientes de variación son significantemente mayores que aquéllos asociados con la incertidumbre en las propiedades de los materiales y variables geométricas en los sistemas estructurales, como puede consultarse en el estudio de Ellingwood et al. (1980). También se observan valores de $v_{\mathrm{Y}}$ mayores a 3.0, pero sólo para sitios asociados con valores pequeños de $m_{\mathrm{Y}}$. El rango de valores de $v_{\mathrm{Y}}$ se reduce cuando $T_{\mathrm{n}}$ se incrementa. Debido a que $v_{\mathrm{Y}}$ no es constante para la aceleración máxima del terreno o la SA (para un valor específico de $T_{\mathrm{n}}$ ) en la región considerada, las tendencias en $y_{2475}$ siguen sólo aproximadamente aquéllas de la media.

Para apreciar mejor el peligro sísmico en función de $T_{\mathrm{n}}$ para el caso considerado en la figura 3 (únicamente sismos interplaca), en la figura 6 se muestran las curvas de los EPU para un periodo de retorno de 2475 años para cuatro sitios seleccionados, los cuales se denotan como Sitios I, II, III y IV, con latitudes y longitudes iguales a $\left(19.5^{\circ},-101^{\circ}\right),\left(17^{\circ},-100^{\circ}\right),\left(17^{\circ},-96.5^{\circ}\right)$ y $\left(16.5^{\circ},-95^{\circ}\right)$, correspondientes aproximadamente a Morelia, Acapulco, Oaxaca y Salina Cruz, respectivamente (ver figura 1a).

Los resultados indican que para sismos interplaca, conforme el sitio se localiza más lejos de la costa, el peligro sísmico disminuye, y que el peligro sísmico para el Sitio II, el cual es el más cercano a la costa, es significativamente más grande que para los otros sitios. Resultados para otros períodos de retorno, no presentados aquí, exhiben tendencias similares.

Si bien el análisis de la desagregación basado en la magnitud, la distancia, y el error de modelación (i.e., $\varepsilon$ en las ecs. 5 a 7) está fuera de los alcances de este estudio, los resultados mostrados en la figura 6 , de hecho, representan la desagregación del peligro sísmico basado en dos diferentes tipos de sismos. Tal desagregación es de interés, ya que los datos estadísticos de la demanda de ductilidad de desplazamiento pueden diferir y afectar la confiabilidad estructural (ver la sección RESPUESTA INELÁSTICA Y CONFIABILIDAD).

Como era de esperarse, para sitios cercanos al centro de las zonas que originan sismos inslab y mostradas en la figura 1c, se tienen los mayores valores de $m_{\mathrm{Y}}$ y $y_{2475}$, como se aprecia en la figura 4 . En contraste con los resultados mostrados en la figura 3 , los $v_{\mathrm{Y}}$ para sismos inslab varían de 5 a 15 para sitios con peligro símico significativo, valores que son mucho más grandes que para el caso de sismos interplaca. El valor de $y_{2475}$ sobre las zonas sísmicas es relativamente consistente y similar a, o mayor que, aquéllos observados para los sismos interplaca si $T_{\mathrm{n}}$ es aproximadamente menor a 0.5 (s). Esto implica que el peligro sísmico debido a sismos inslab puede regir el diseño estructural para estructuras rígidas, una observación que ya había sido advertida por Jaimes y Reinoso (2006). Sin embargo, conforme $T_{\mathrm{n}}$ es aproximadamente mayor que 0.5 (s), los valores de $y_{2475}$, en sitios sobre las zonas sísmicas consideradas, son comparables a aquéllos debidos a sismos interplaca. Para investigar aún más la contribución relativa de los sismos inslab al peligro sísmico de la región, el EPU para un periodo de retorno igual a 2475 años se grafica en la figura 6 para los sitios mencionados anteriormente, y se compara con aquéllos debidos a sismos interplaca.

En la figura 6 también se observa que el peligro sísmico para sitios cercanos a Morelia y Oaxaca debido a sismos inslab, es significativamente mayor que aquél debido a sismos interplaca; el peligro causado por sismos inslab para el sitio cercano a Acapulco, no es significativo comparado con el causado por sismos interplaca, en tanto que para el sitio cercano a Salina Cruz, el peligro debido a sismos inslab es comparable a aquél debido a sismos interplaca. 

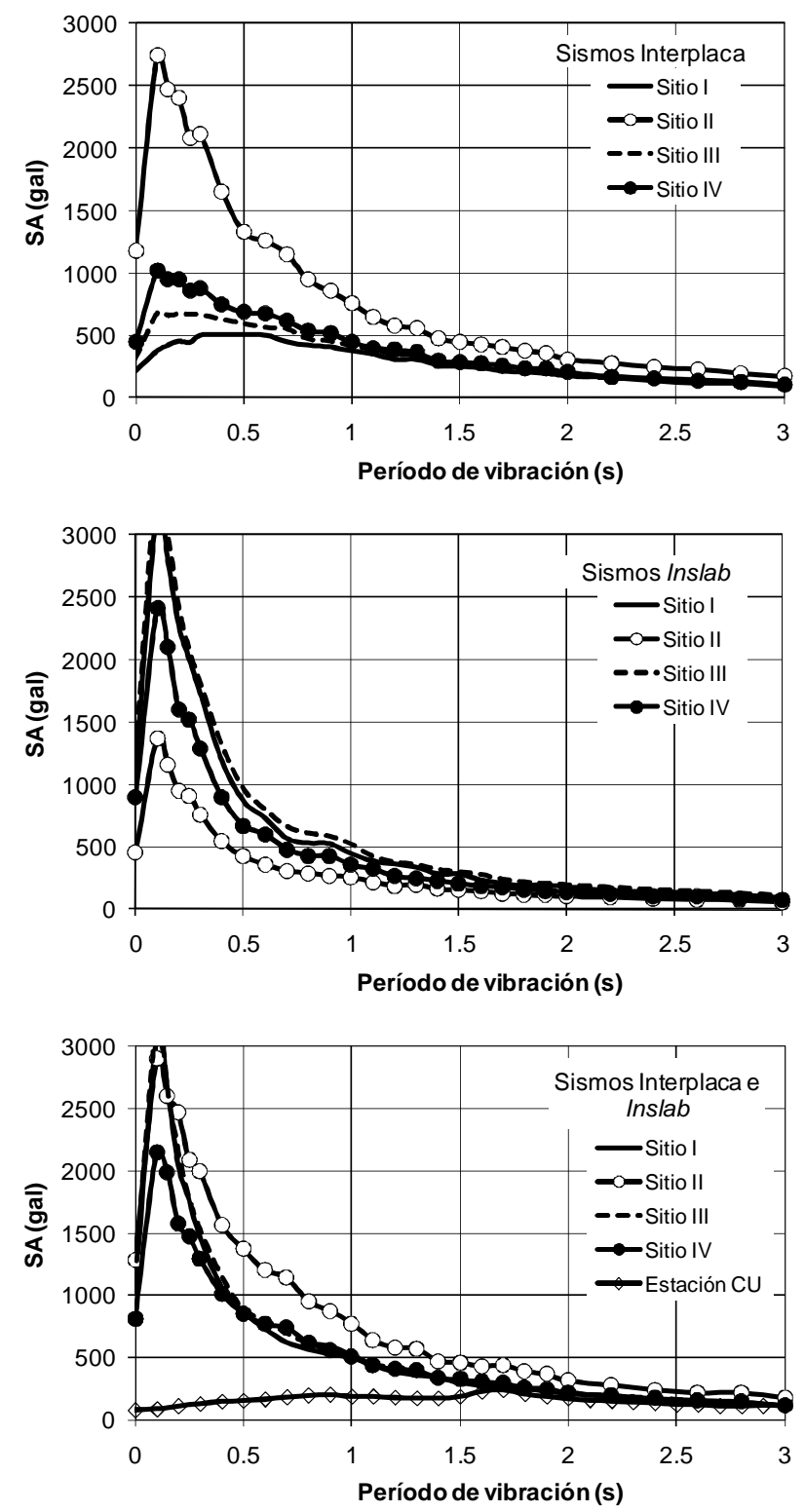

Figura 6. Espectros de peligro uniforme con base en un período de retorno de 2475 años para los cuatro sitios seleccionados y la estación CU

Los mapas de contorno mostrados en la figura 5, asociados a sismos inslab e interplaca, exhiben peligros más grandes que aquéllos mostrados en las figuras 3 y 4 , lo que también era de esperarse. Los valores de $v_{\mathrm{Y}}$ se reducen significativamente, si se comparan con aquéllos causados por los sismos inslab, y se incrementan, si la comparación se realiza con los coeficientes de variación causados por sismos interplaca. En general, los $v_{\mathrm{Y}}$ para sismos interplaca e inslab varían de 4 a 7 para sitios con peligro sísmico considerable y para $T_{\mathrm{n}}$ menor que aproximadamente $0.5(\mathrm{~s})$; el rango de valores de $v_{\mathrm{Y}}$ se reduce para $T_{\mathrm{n}}$ mayor que $0.5(\mathrm{~s})$. En particular, si $T_{\mathrm{n}}$ es mayor que aproximadamente $1.0(\mathrm{~s}), v_{\mathrm{Y}}$ fluctúa entre $2 \mathrm{y}$ 3 para sitios con peligro sísmico importante. Una vez más, los EPU debidos conjuntamente a sismos interplaca e inslab se muestran en la figura 6 con fines comparativos para los sitios seleccionados. Los 
EPU debidos a ambos tipos de sismo, son una envolvente de los debidos a un particular tipo de sismo (interplaca o inslab); la forma de los EPU depende de si el peligro es dominado por sismos interplaca o inslab.

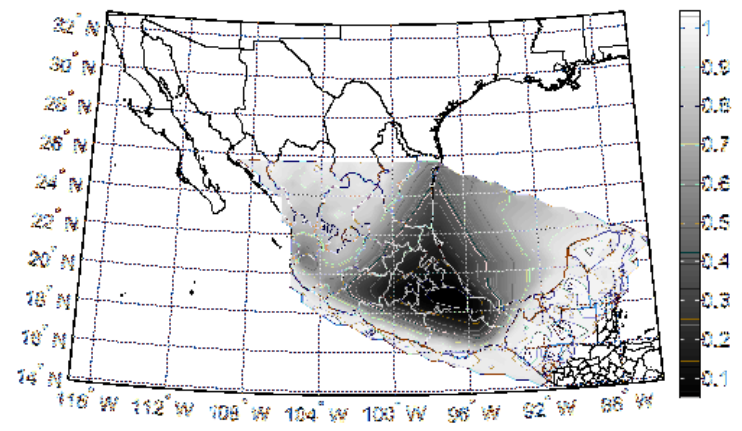

a1) Cocientes para interplaca y $\mathrm{SA}\left(T_{\mathrm{n}}=0 \mathrm{~s}\right)$

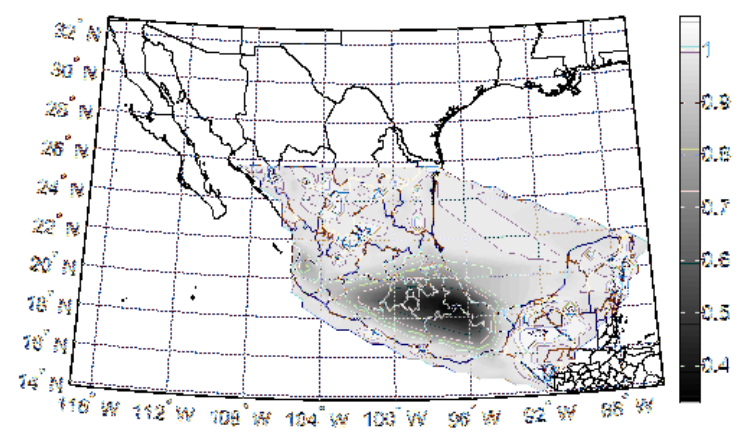

a2) Cocientes para interplaca y SA $\left(T_{\mathrm{n}}=0.5 \mathrm{~s}\right)$

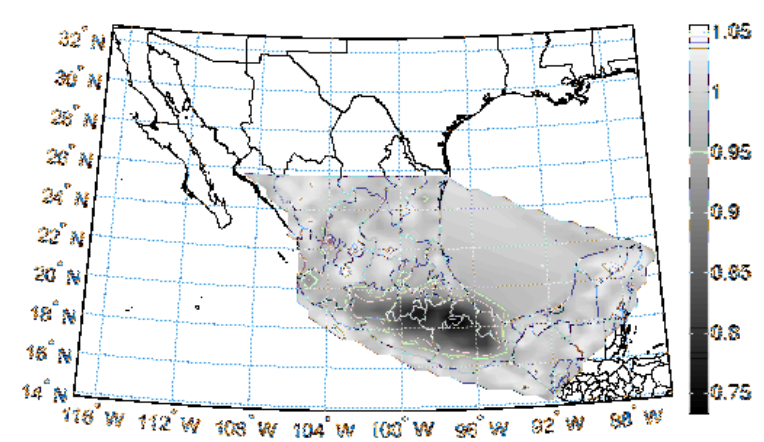

a3) Cocientes para interplaca y $\mathrm{SA}\left(T_{\mathrm{n}}=2 \mathrm{~s}\right)$

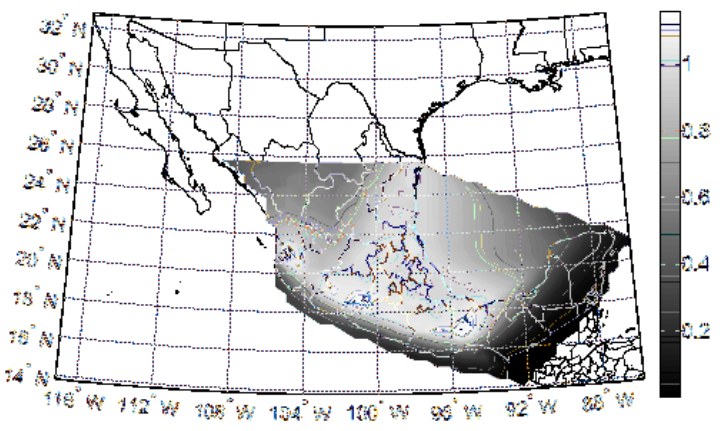

b1) Cocientes para inslab y SA $\left(T_{\mathrm{n}}=0 \mathrm{~s}\right)$

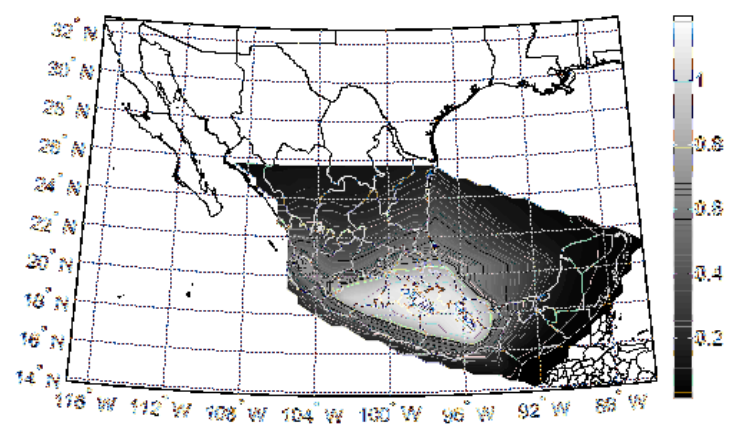

b2) Cocientes para inslab y SA $\left(T_{\mathrm{n}}=0.5 \mathrm{~s}\right)$

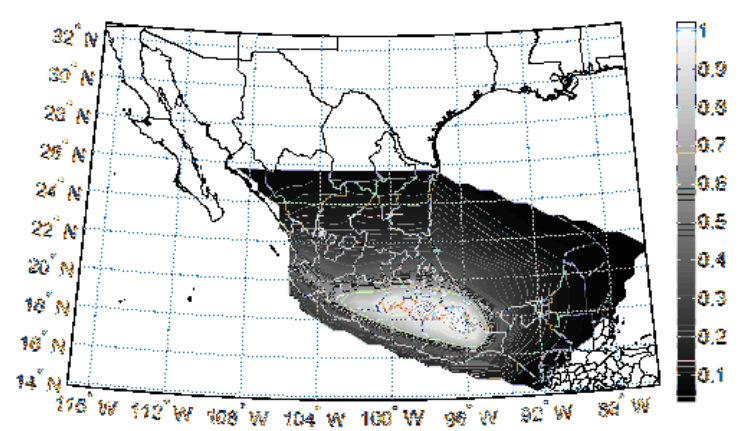

b3) Cocientes para inslab y SA $\left(T_{\mathrm{n}}=2 \mathrm{~s}\right)$

Figura 7. Mapas de contorno de cocientes: los conjuntos a1), a2) y a3); y b1), b2) y b3); corresponden a $y_{2475}$ de sismos interplaca e inslab, respectivamente, entre $y_{2475}$ de sismos conjuntos interplaca e inslab

Con fines comparativos, también se incluye en la figura 6 el EPU para la estación CU (localizada en la UNAM en la Ciudad de México), el cual se calculó utilizando las zonas sísmicas mostradas en la tabla 1 para sismos interplaca y la ec. 5. La figura muestra que la forma del EPU para la estación de CU es significativamente diferente que para los sitios cercanos a la región de la costa del Pacífico Mexicano, y que las magnitudes del EPU son comparables a aquéllas para la zona costera solamente para estructuras 
con periodos de vibración largos. Cabe mencionar que Singh et al. (1995) señalaron las diferencias de los efectos sísmicos en el valle de México y en la zona de la costa del Pacífico para algunos sismos históricos individuales.

Para cuantificar aún más las contribuciones relativas de los sismos interplaca e inslab al peligro sísmico, se evalúan los cocientes de $y_{2475}$ de los resultados mostrados en las figuras 3 ó 4 en relación a aquéllos mostrados en la figura 5. Tales cocientes se grafican en la figura 7, en la que se observa que la contribución de los sismos interplaca es más relevante cerca de la costa, como era de suponerse, y que en general, se vuelve más significativa para valores grandes de $T_{\mathrm{n}}$.

Un aspecto más que merece estudiarse, es la distribución de probabilidad de la SA. Para ello se extrajeron muestras de las simulaciones de la SA máxima anual y se presentan en la figura 8 (como gráficas de probabilidad lognormal), para el caso de eventos interplaca e inslab contribuyendo conjuntamente al peligro sísmico. En todos los casos, las gráficas muestran que la SA puede ser modelada como una variable lognormal, por lo menos en el extremo superior de la distribución de probabilidad. La suposición de que $Y$ es una variable aleatoria lognormal, es común en el análisis de confiabilidad de estructuras sujetas a excitaciones sísmicas.
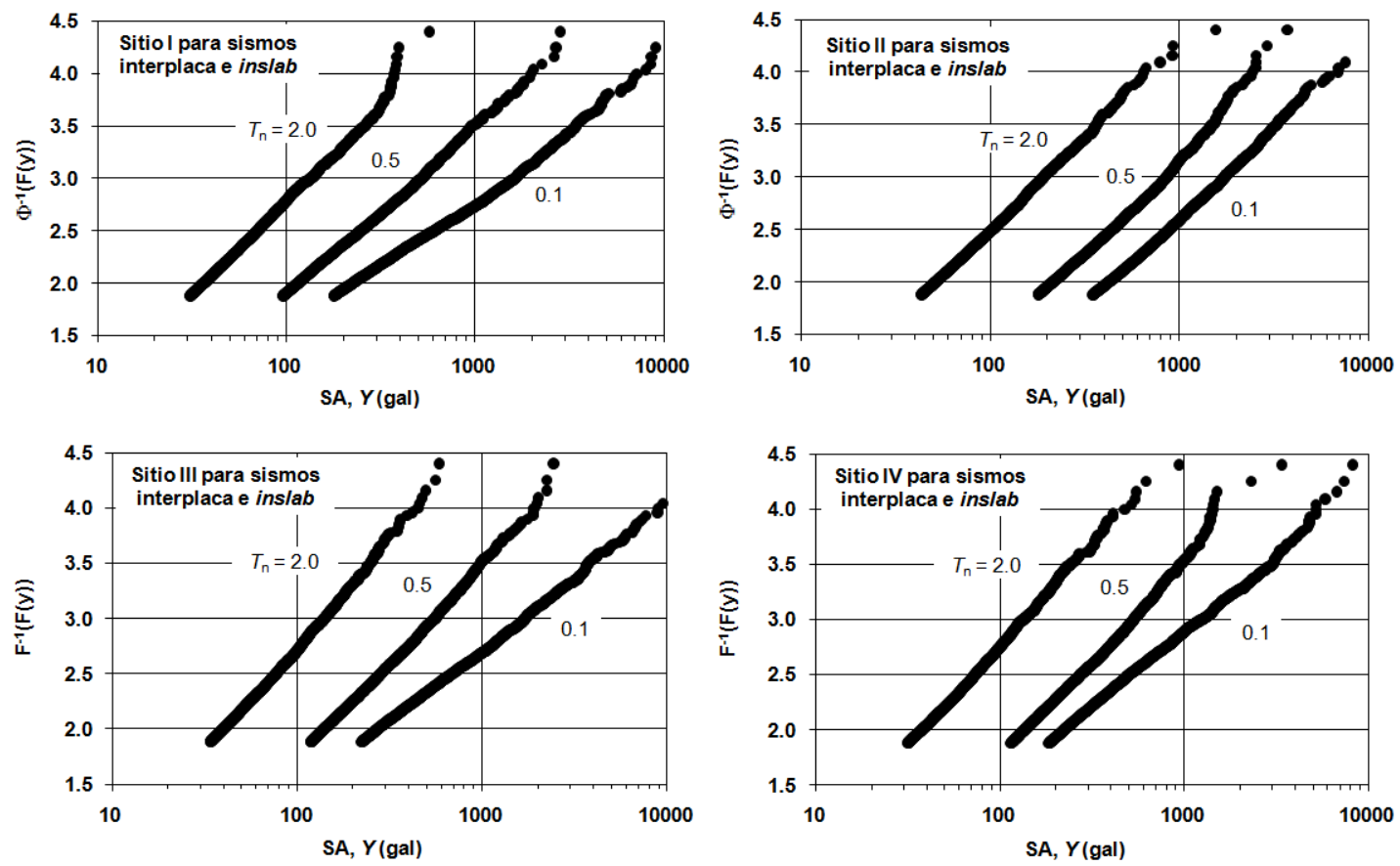

Figura 8. Distribuciones de probabilidad empíricas de $Y$ se muestran en graficas de probabilidad lognormal

\section{RESPUESTA INELÁSTICA Y CONFIABILIDAD}

\section{Demanda de ductilidad de desplazamiento}

Los edificios y puentes se diseñan para incurrir en deformaciones inelásticas debidas a grandes sismos. En el actual reglamento de puentes (SCT 2001) el coeficiente sísmico basal $C_{\mathrm{s}}$ es igual a $a$ 
dividido por $Q^{\prime}$, mientras que en el reglamento de la Ciudad de México (NTCS 2004) se considera un término adicional debido a la sobreresistencia $R_{0}$; en dichos códigos, $a$ es el coeficiente sísmico elástico de diseño y $Q^{\prime}$ es un factor de reducción que depende de $T_{\mathrm{n}}$ y del factor de comportamiento sísmico, $Q$, cuya cuantificación y significado físico no son del todo claros. De hecho, Rosenblueth y Gómez (1991) subrayaron que $Q$ no es la capacidad de ductilidad de desplazamiento, y que por mucho equivaldría a alrededor del $40 \%$ de dicha capacidad. Por lo tanto, podría ser instructivo reemplazar $Q^{\prime}$ por un factor de reducción relacionado con la capacidad de ductilidad de desplazamiento $R_{\mu}$, tal como fue considerado en el más reciente MOC (2008). No obstante, a diferencia del caso del MOC (2008), en este estudio se considera que el cociente definido por la media de la fuerza cortante basal entre la mínima fuerza cortante basal de diseño requerida, $R_{\mathrm{o}}$, incluye el efecto del comportamiento del sistema, así como la diferencia entre la media de la resistencia del material y su resistencia de diseño, y la diferencia entre los tamaños de los miembros estructurales diseñados y los tamaños requeridos dictados por los requerimientos mínimos de diseño. Además, se considera que el uso de una forma constante del espectro elástico de diseño para $a$ podría no ser apropiado para una región amplia de México, ya que tal espectro no garantiza una probabilidad de excedencia consistente para sitios localizados dentro de dicha región, la que podría ser afectada por diferentes tipos de sismo con características de excitación distintas como se estudió en la sección anterior.

Con base en lo arriba expuesto, y considerando que la estructura diseñada puede ser modelada como un sistema histerético bilineal de un grado de libertad cuyo diseño está regido por el criterio de resistencia y sujeto a la demanda sísmica $S\left(T_{\mathrm{n}}, \xi\right)(\mathrm{g})$, la resistencia de fluencia normalizada $\phi$ puede ser expresada como,

$$
\phi=\min (\zeta, 1)
$$

donde

$$
\zeta=\frac{S_{E}\left(T_{n}, \xi\right)}{S\left(T_{n}, \xi\right)} \frac{R_{n}}{R_{o} R_{\mu}}
$$

y donde $R_{\mathrm{n}}$ denota el cociente de la capacidad de fluencia de la estructura diseñada entre la fuerza cortante basal mínima de diseño, y $R_{\mu}$ es un factor de reducción relacionado con la ductilidad. El factor de irregularidad y el de importancia de la estructura se ignoran en las ecs. 9.

Para un valor dado o estimado de $\phi$, se dispone de los datos estadísticos de la demanda de ductilidad para los grupos de registros sísmicos aquí considerados y para distintos valores del cociente de la rigidez de postfluencia entre la rigidez inicial, $\gamma$ (Pozos-Estrada et al. 2008, Hong et al. 2010), por lo que la media de la demanda de ductilidad de desplazamiento $\mu$, denotada por $m_{\mu}$, puede ser aproximada con,

$m_{\mu}=\exp \left(\left(-\alpha_{1} \ln \phi\right)^{b}\right)$

donde los parámetros de modelación $\alpha_{1}$ y $b$, también están disponibles en los estudios mencionados y como complemento se reproducen en la tabla 3. El cov de $\mu, v_{\mu}$, es una función decreciente de $\phi$, excepción hecha para estructuras muy rígidas; en la mayoría de los casos $v_{\mu}$ varía aproximadamente entre 0.4 y 1.2 para $\phi$ entre 0.1 y 0.5 . Gráficas ilustrando la variación de $m_{\mu}$ y $v_{\mu}$ se muestran en las figuras 9 y 10 , respectivamente. 
Tabla 3. Parámetros de modelación para la demanda de ductilidad empírica determinada por la ec. 10

\begin{tabular}{|c|c|c|c|c|c|c|c|c|c|}
\hline \multirow[b]{2}{*}{ Caso } & \multirow[b]{2}{*}{$T_{\mathrm{n}}$} & \multicolumn{2}{|c|}{$\gamma=0$} & \multicolumn{2}{|c|}{$\gamma=0.01$} & \multicolumn{2}{|c|}{$\gamma=0.05$} & \multicolumn{2}{|c|}{$\gamma=0.1$} \\
\hline & & $\alpha_{1}$ & $b$ & $\alpha_{1}$ & $b$ & $\alpha_{1}$ & $b$ & $\alpha_{1}$ & $b$ \\
\hline \multirow[t]{9}{*}{ I } & 0.1 & 7.117 & 0.740 & 7.127 & 0.902 & 5.817 & 0.788 & 4.361 & 0.812 \\
\hline & 0.2 & 3.929 & 1.087 & 3.252 & 1.261 & 2.531 & 1.058 & 2.183 & 1.068 \\
\hline & 0.3 & 2.902 & 1.290 & 2.519 & 1.170 & 2.290 & 1.076 & 1.985 & 1.105 \\
\hline & 0.4 & 2.384 & 1.327 & 2.160 & 1.098 & 2.014 & 1.031 & 1.800 & 1.062 \\
\hline & 0.5 & 2.066 & 1.355 & 2.039 & 1.021 & 1.846 & 1.011 & 1.666 & 1.054 \\
\hline & 0.7 & 1.689 & 1.398 & 2.002 & 0.943 & 1.700 & 1.022 & 1.557 & 1.073 \\
\hline & 1.0 & 1.388 & 1.444 & 1.681 & 0.973 & 1.477 & 1.034 & 1.354 & 1.103 \\
\hline & 2.0 & 0.909 & 1.532 & 1.505 & 0.998 & 1.319 & 1.064 & 1.221 & 1.148 \\
\hline & 3.0 & 1.040 & 1.584 & 1.330 & 1.047 & 1.196 & 1.094 & 1.105 & 1.196 \\
\hline \multirow[t]{9}{*}{ II } & 0.1 & 2.561 & 1.207 & 2.433 & 1.108 & 2.115 & 1.074 & 1.870 & 1.038 \\
\hline & 0.2 & 1.557 & 1.193 & 1.531 & 1.085 & 1.371 & 1.143 & 1.273 & 1.169 \\
\hline & 0.3 & 1.284 & 1.188 & 1.263 & 1.083 & 1.139 & 1.151 & 1.076 & 1.196 \\
\hline & 0.4 & 1.205 & 1.127 & 1.158 & 1.084 & 1.073 & 1.105 & 1.022 & 1.156 \\
\hline & 0.5 & 1.121 & 1.119 & 1.105 & 1.028 & 1.002 & 1.094 & 0.954 & 1.154 \\
\hline & 0.7 & 1.064 & 1.058 & 1.027 & 1.018 & 0.937 & 1.064 & 0.892 & 1.127 \\
\hline & 1.0 & 1.011 & 1.055 & 0.990 & 0.996 & 0.893 & 1.057 & 0.852 & 1.111 \\
\hline & 2.0 & 0.995 & 1.034 & 0.975 & 0.986 & 0.884 & 1.054 & 0.845 & 1.095 \\
\hline & 3.0 & 1.054 & 0.986 & 1.037 & 0.947 & 0.943 & 1.016 & 0.902 & 1.063 \\
\hline \multirow[t]{9}{*}{ III } & 0.1 & 1.831 & 1.256 & 1.724 & 1.169 & 1.479 & 1.178 & 1.338 & 1.160 \\
\hline & 0.2 & 1.384 & 1.214 & 1.313 & 1.144 & 1.162 & 1.177 & 1.077 & 1.207 \\
\hline & 0.3 & 1.254 & 1.202 & 1.192 & 1.129 & 1.068 & 1.171 & 1.003 & 1.217 \\
\hline & 0.4 & 1.202 & 1.143 & 1.129 & 1.107 & 1.010 & 1.156 & 0.951 & 1.209 \\
\hline & 0.5 & 1.153 & 1.140 & 1.114 & 1.054 & 0.985 & 1.131 & 0.928 & 1.197 \\
\hline & 0.7 & 1.144 & 1.053 & 1.082 & 1.020 & 0.960 & 1.090 & 0.909 & 1.142 \\
\hline & 1.0 & 1.070 & 1.058 & 1.018 & 1.027 & 0.903 & 1.117 & 0.857 & 1.182 \\
\hline & 2.0 & 1.081 & 0.991 & 1.044 & 0.943 & 0.916 & 1.041 & 0.869 & 1.103 \\
\hline & 3.0 & 1.012 & 1.004 & 0.988 & 0.954 & 0.889 & 1.030 & 0.850 & 1.086 \\
\hline
\end{tabular}

Nota: Los Casos I, II y III representan los parámetros de modelación para la estación CU (Pozos-Estrada et al. 2008), sismos interplaca, y sismos inslab (Hong et al. 2010), respectivamente.

De acuerdo a los estudios referidos en las líneas anteriores, en términos generales, el modelo de probabilidad de Frechet puede ser adecuado para $\mu$, excepto para $T_{\mathrm{n}}<0.3$, en cuyo caso se prefiere el modelo de probabilidad lognormal. Una de las observaciones principales de estos estudios, es que en general los datos estadísticos de la demanda de ductilidad de desplazamiento difieren para los sismos Mexicanos inslab e interplaca. Tal diferencia requiere ser incorporada en el análisis de confiabilidad y en la calibración de reglamentos de diseño.

Cabe mencionar que la ec. 10 es válida para una orientación aleatoria, debido a que análisis estadísticos muestran que las orientaciones de los registros no afectan a $\mu$ (García Soto et al. 2012). 

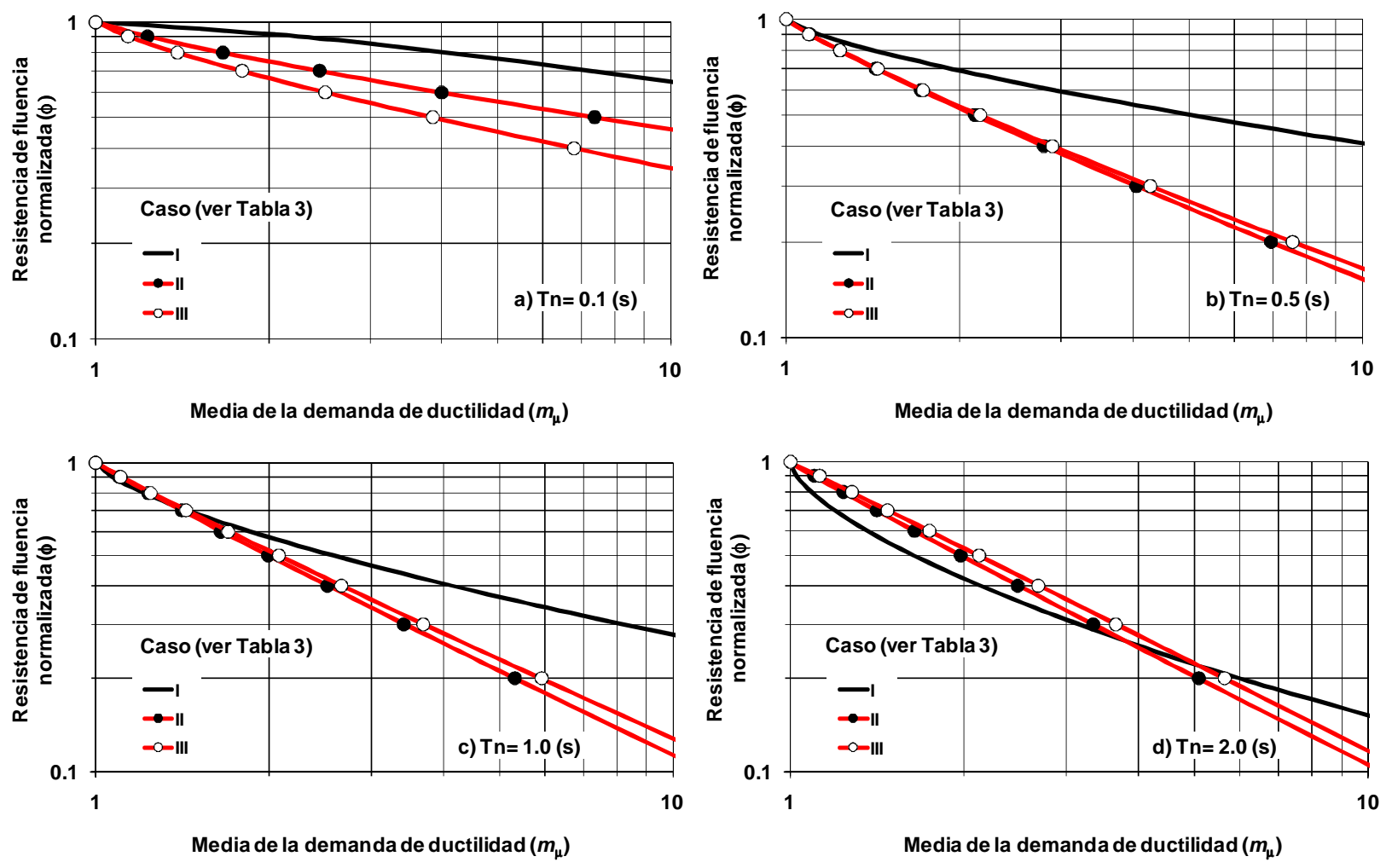

Figura 9. Media de la demanda de ductilidad para la estación CU (Caso I), y sismos interplaca (Caso II) e inslab (Caso III)

\section{Cálculo de confiabilidad e implicación en el reglamento de diseño}

El análisis de confiabilidad se simplifica si se aproxima el comportamiento de las estructuras como sistemas bilineales histeréticos de un grado de libertad. La función de estado límite para el colapso incipiente, $g_{c}$, puede expresarse como,

$g_{C}=\mu_{R} / \mu(\mathbf{A})-1$,

donde $\mu_{\mathrm{R}}$ denota la capacidad de ductilidad del sistema, y $\mu$, la demanda de ductilidad de desplazamiento, se remplaza por $\mu(\mathbf{A})$ para resaltar que ésta depende o es función de $\mathbf{A}, \mathbf{A}=\left[\phi, \gamma, T_{\mathrm{n}}, \xi\right]$. La combinación de valores de $\phi$ menores que 1.0 y $g_{c}$ menores que cero implica colapso incipiente.

Antes de llevar a cabo el estudio paramétrico de la probabilidad anual de que $g_{c} \leq 0$, denotada por $P_{\mathrm{c}}$, señalamos que $R_{\mathrm{n}} / R_{\mathrm{o}}$ (que representa la capacidad (real) de fluencia entre la capacidad media del sistema) debe ser, en promedio, mayor que 1.0 para el sistema, y que la incertidumbre en $R_{\mathrm{n}} / R_{\mathrm{o}}$ puede ignorarse dado que su impacto en la $P_{\mathrm{c}}$ estimada es usualmente despreciable. Siguiendo la práctica actual en otras jurisdicciones en el mundo y sin pérdida de generalidad, por el momento $S_{E}\left(T_{\mathrm{n}}, \xi\right)$ se iguala con el valor correspondiente a un periodo de retorno de 2475 años. $\mu_{\mathrm{R}}$ puede modelarse como una variable aleatoria lognormal (Díaz-López y Esteva 1991) con un valor de cov, $v_{\mu_{R}}$, variando de 0.5 a 1.0. Lo anterior es orientado por el cov de la ductilidad de miembros de acero que varía de 0.5 a 1.0 (Nakashima 1997). 


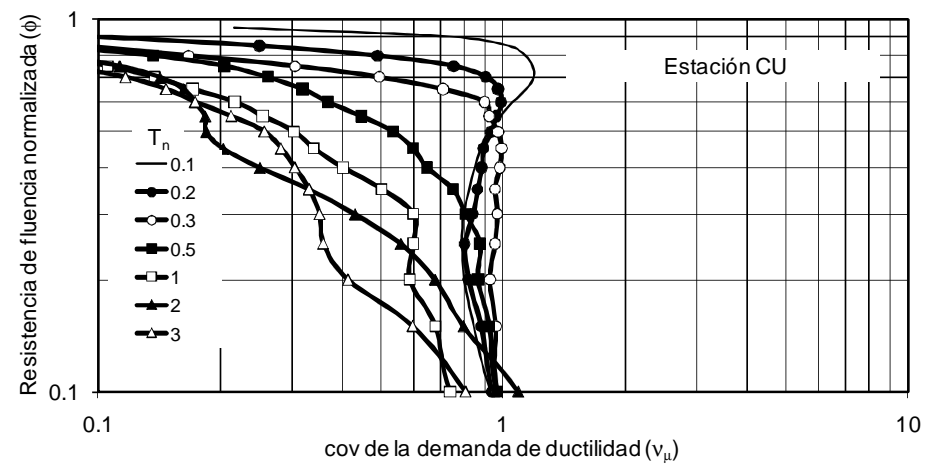

(a)

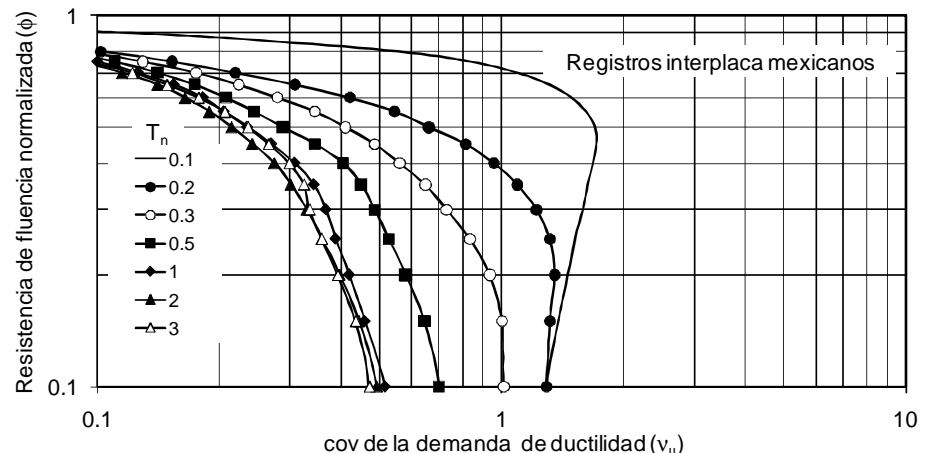

(b)

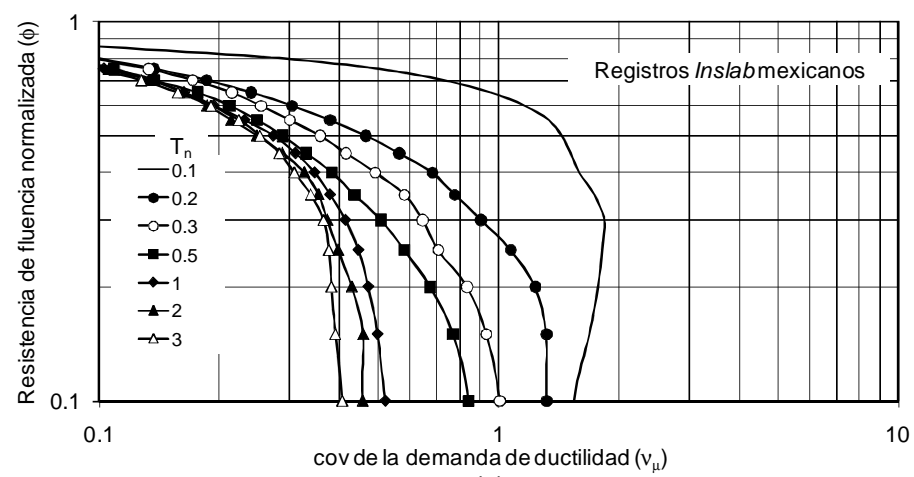

(c)

Figura 10. Coeficientes de variación de la demanda de ductilidad para la estación CU, y para sismos interplaca e inslab

Se asume que el factor de reducción relacionado con la capacidad de ductilidad de desplazamiento $R_{\mu}$, es igual a la media de $\mu_{\mathrm{R}}, m_{\mu_{R}}$. Ya que $\mu(\mathbf{A})$ difiere para sismos interplaca e inslab, $P_{\mathrm{c}}$ requiere estimarse sumando la probabilidad de colapso incipiente debido a sismos interplaca, más la probabilidad de colapso incipiente debido a sismos inslab.

Considérese que $S_{i}\left(T_{n}, \xi\right)$ denota la $\mathrm{SA}$ originada por el $i$-ésimo tipo de sismo, $i=1$ y 2 representa respectivamente los sismos interplaca e inslab. Para estimar $P_{\mathrm{c}}$, se emplea la técnica de simulación (Hong y Hong 2007), donde $P_{\mathrm{c}}$ representa la suma de la probabilidad de colapso incipiente debido a cada tipo de 
sismo. Básicamente, se simula un valor de $S_{i}\left(T_{n}, \xi\right)$ y se le utiliza en la ec. 9 para evaluar $\phi$; se generan muestras de $\mu_{\mathrm{R}}$ y $\mu(\mathbf{A})$ y se usan en la ec. 11 para evaluar $g$ cuando $\phi$ es menor que 1.0. Nótese que los valores estadísticos de $S_{i}\left(T_{n}, \xi\right)$ para un sitio específico, pueden obtenerse de resultados similares a los mostrados en las figuras 3 a 5 (ver también la tabla 4 para unos cuantos sitios seleccionados).

Tabla 4. Modelos probabilísticos de peligro sísmico para los sitios considerados que se muestran en la

\begin{tabular}{|c|c|c|c|c|c|c|c|c|c|c|c|c|c|c|c|}
\hline \multirow{3}{*}{$\begin{array}{c}\begin{array}{c}\text { Período } \\
\text { (s) }\end{array} \\
0.1\end{array}$} & \multicolumn{3}{|c|}{$\begin{array}{l}\text { Sitio I }\left(19.5^{\circ},-101^{\circ}\right) \\
\text { Cerca de Morelia }\end{array}$} & \multicolumn{3}{|c|}{$\begin{array}{l}\text { Sitio II }=\left(17^{\circ},-100^{\circ}\right) \\
\text { Cerca de Acapulco }\end{array}$} & \multicolumn{3}{|c|}{$\begin{array}{l}\text { Sitio III }\left(17^{\circ},-96.5^{\circ}\right) \\
\text { Cerca de Oaxaca }\end{array}$} & \multicolumn{3}{|c|}{$\begin{array}{c}\text { Sitio IV }\left(16.5^{\circ},-95^{\circ}\right) \\
\text { Cerca de Salina } \\
\text { Cruz }\end{array}$} & \multicolumn{3}{|c|}{$\begin{array}{c}\text { Sitio V } \\
\text { Estación CU }\end{array}$} \\
\hline & $m_{\mathrm{S}}$ & $v_{\mathrm{S}}$ & $S_{E}\left(T_{\mathrm{n}}, \xi\right)$ & $m_{\mathrm{S}}$ & $v_{\mathrm{S}}$ & $S_{E}\left(T_{\mathrm{n}}, \xi\right)$ & $m_{\mathrm{S}}$ & $v_{\mathrm{S}}$ & $S_{E}\left(T_{\mathrm{n}}, \xi\right)$ & $m_{\mathrm{S}}$ & $v_{\mathrm{S}}$ & $S_{E}\left(T_{\mathrm{n}}, \xi\right)$ & $m_{\mathrm{S}}$ & $v_{\mathrm{S}}$ & $S_{E}\left(T_{\mathrm{n}}, \xi\right)$ \\
\hline & 9.7 & 2.36 & 373.9 & 58.2 & 2.79 & 2739.5 & 18.8 & 2.23 & 677.1 & 25.5 & 2.42 & 1016.8 & & & \\
\hline & 28.7 & 7.56 & 3226.4 & 16.1 & 5.19 & 1372.6 & 33.1 & 6.90 & 3497.0 & 15.4 & 13.94 & 2415.0 & 7.0 & 1.04 & 86.0 \\
\hline & 30.6 & 6.40 & 3066.7 & 68.2 & 2.56 & 2908.9 & 37.7 & 5.15 & 3205.5 & 31.4 & 4.02 & 2152.9 & & & \\
\hline \multirow[t]{3}{*}{0.2} & 11.9 & 2.31 & 449.3 & 54.3 & 2.64 & 2399.0 & 20.5 & 2.07 & 673.4 & 25.8 & 2.26 & 944.3 & & & \\
\hline & 19.7 & 7.93 & 2281.2 & 11.7 & 4.89 & 949.3 & 23.0 & 6.90 & 2428.9 & 10.7 & 12.61 & 1601.0 & 9.3 & 1.04 & 112.4 \\
\hline & 23.9 & 5.23 & 2051.4 & 59.5 & 2.50 & 2470.7 & 30.9 & 4.11 & 2161.4 & 29.5 & 3.12 & 1571.8 & & & \\
\hline \multirow{3}{*}{0.5} & 13.4 & 2.29 & 501.3 & 32.2 & 2.48 & 1325.8 & 18.4 & 2.05 & 596.6 & 20.4 & 2.10 & 683.1 & & & \\
\hline & 8.4 & 6.71 & 870.0 & 5.6 & 4.43 & 422.0 & 9.6 & 6.40 & 966.8 & 4.6 & 11.96 & 671.1 & 11.2 & 1.15 & 159.8 \\
\hline & 17.8 & 2.88 & 865.5 & 35.7 & 2.35 & 1374.1 & 23.4 & 2.33 & 891.1 & 22.6 & 2.32 & 855.5 & & & \\
\hline \multirow[t]{3}{*}{1.0} & 11.4 & 2.06 & 370.6 & 18.7 & 2.45 & 757.0 & 13.1 & 2.02 & 415.0 & 13.5 & 2.08 & 446.4 & & & \\
\hline & 4.3 & 6.76 & 446.9 & 3.1 & 4.96 & 257.1 & 4.9 & 7.11 & 522.8 & 2.4 & 12.40 & 354.6 & 13.0 & 1.21 & 198.8 \\
\hline & 13.5 & 2.28 & 502.0 & 19.4 & 2.42 & 775.1 & 16.1 & 2.05 & 521.9 & 14.6 & 2.16 & 506.3 & & & \\
\hline \multirow[t]{3}{*}{2.0} & 5.6 & 1.98 & 172.5 & 8.3 & 2.30 & 308.6 & 6.3 & 1.87 & 179.7 & 5.9 & 2.14 & 202.7 & & & \\
\hline & 1.6 & 7.37 & 179.3 & 1.3 & 4.93 & 102.8 & 1.8 & 7.20 & 196.5 & 0.9 & 12.80 & 139.5 & 12.1 & 1.18 & 180.0 \\
\hline & 6.4 & 2.07 & 208.2 & 8.7 & 2.23 & 313.5 & 7.2 & 1.96 & 219.4 & 6.6 & 2.07 & 215.6 & & & \\
\hline
\end{tabular}

Para los sitios considerados, mostrados en la figura 1a, incluyendo la estación CU en la Ciudad de México, y cuyos modelos probabilísticos de la SA se enlistan en la tabla 4, los valores estimados de $P_{\mathrm{c}}$ se muestran en la figura 11 para $R_{\mu}=m_{\mu_{R}}$, y combinaciones de $R_{\mathrm{n}} / R_{\mathrm{o}}=1.15, m_{\mu_{R}}=2 \mathrm{y} 4, v_{\mu_{R}}=0.5 \mathrm{y} 1.0, \mathrm{y}$ $T_{\mathrm{n}}=0.1,0.2,0.5,1$ y 2 . Los resultados mostrados en la figura y otros no incluidos aquí para ahorrar espacio, sugieren que:

1) Para los Sitios I a IV, el uso consistente de valores de $S_{E}\left(T_{n}, \xi\right)$ para un período de retorno dado, conlleva a su vez a valores consistentes de $P_{\mathrm{c}}$ para cocientes fijos de $R_{\mathrm{n}} / R_{\mathrm{o}} . P_{\mathrm{c}}$ depende de $R_{\mathrm{n}} / R_{\mathrm{o}}$ como se esperaba, por lo tanto, si se desea aun más consistencia en $P_{\mathrm{c}}$, deben hacerse recomendaciones de tal manera que $R_{\mathrm{n}} / R_{\mathrm{o}}$ sea uniforme para un rango de casos de diseño.

2) $P_{\mathrm{c}}$ para la estación CU es marcadamente diferente que aquéllas para los Sitios I a IV. Esto puede explicarse al notar que la media de la demanda de ductilidad para la estación de CU difiere significativamente que aquélla para otros Sitios (ver figura 9).

3) Para la estación CU, $P_{\mathrm{c}}$ se incrementa a medida que la media o el cov de $\mu_{\mathrm{R}}$ aumenta.

Esta tendencia en el incremento de $P_{\mathrm{c}}$ para la estación CU, en directa proporción a $m_{\mu_{\mathrm{R}}}$, se explica si notamos que, a medida que $m \mu_{\mathrm{R}}$ aumenta, la $\phi$ debida a los eventos sísmicos probablemente será reducida (ver ec. 9), y un significativo cov de la demanda de ductilidad se asocia con valores más pequeños de $\phi$. Sin embargo, esta clara tendencia no se presenta para los Sitios I a IV, excepto para valores pequeños de $T_{\mathrm{n}} \mathrm{y}$ diferentes $m_{\mu_{\mathrm{R}}}$. Esto se atribuye a las diferencias en las curvas de cov mostradas 
en las figuras 10b y 10c a aquéllas mostradas en la figura 10a. Por lo tanto, para aumentar todavía más la consistencia en la confiabilidad, un valor de $R_{\mu}$ dependiente del período (similar al caso para el Q implementado en el reglamento de la Ciudad de México (NTCS 2004) pero con diferentes parámetros) debería incorporarse. Sin embargo, este aspecto está fuera de los alcances de este estudio.
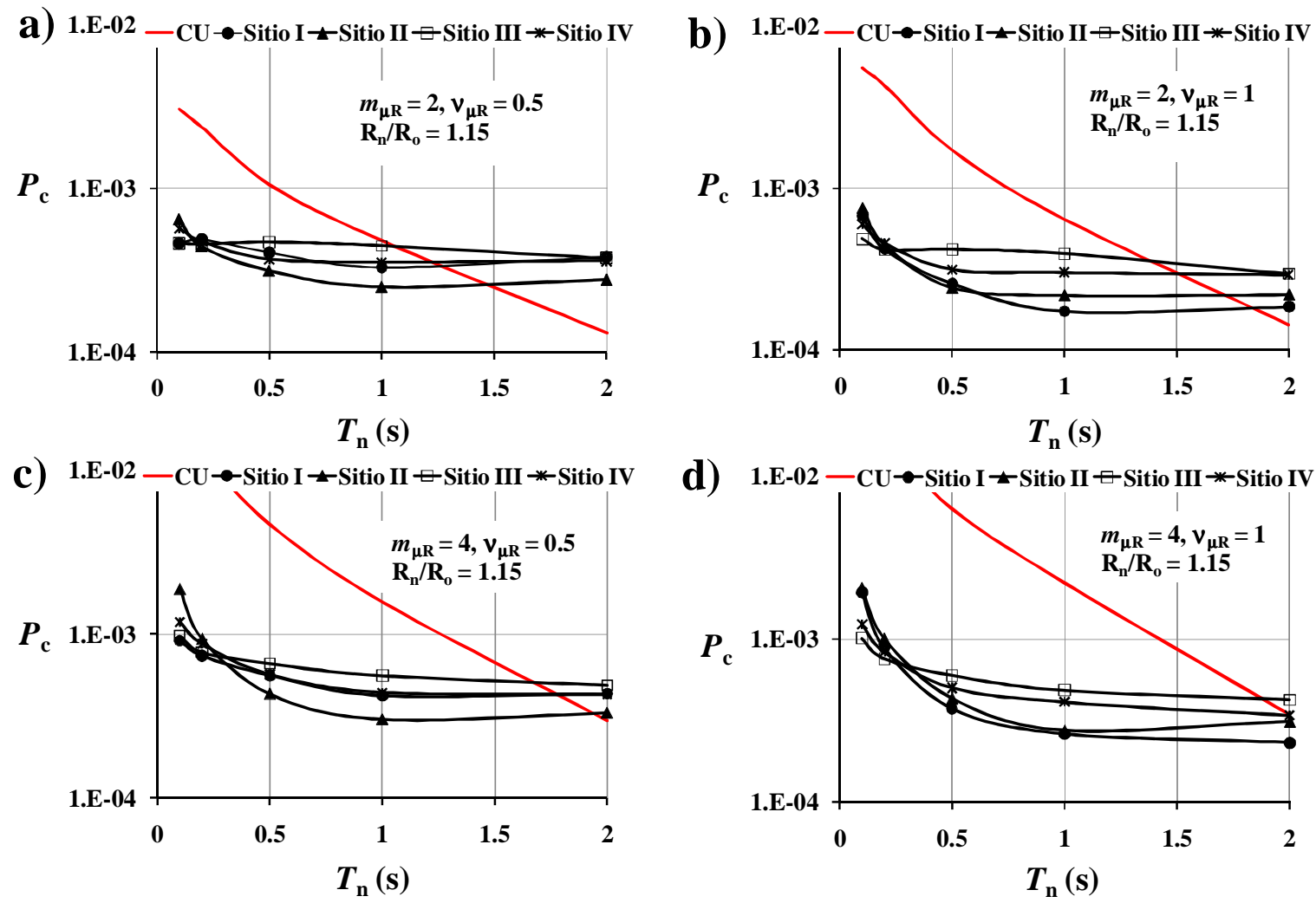

Figura 11. Estimación de la probabilidad anual de colapso incipiente para el formato de diseño considerado y los sitios seleccionados: a) a d)

\section{RESUMEN Y CONCLUSIONES}

Se integró un modelo de riesgo sísmico y fue empleado para evaluar mapas de contorno de peligro uniforme para regiones cercanas a la costa del Pacífico Mexicano que son significativamente afectadas por sismos interplaca e inslab. De los resultados de los mapas de contorno se desprende que, dependiendo del sitio considerado, el peligro sísmico predominante para estructuras rígidas podría provenir principalmente de sismos interplaca, o de sismos inslab. El coeficiente de variación (cov) de la SA varía significativamente con el periodo de vibración, y sus valores están entre aproximadamente 2 a 10 si se consideran ambos tipos de sismos, interplaca e inslab. Este rango de valores de cov es mucho más grande que aquél para la estación CU, el cual es de alrededor de 1.2. Una comparación de los valores estadísticos de la demanda de ductilidad también indica que la media y el cov de la demanda de ductilidad para sitios cerca del área costera son más pequeños que aquéllos para la estación CU. Por lo tanto, los valores estadísticos de peligro sísmico de esta última, no deben aplicarse a la primera (o viceversa). 
$\mathrm{Al}$ adoptar un formato de diseño que es similar al recomendado en el MOC (2008) y el NBCC (2005), se puede obtener un nivel de confiabilidad relativamente consistente si los factores de reducción son apropiadamente seleccionados y la SA (nominal) es seleccionada con base en un periodo de retorno de 2475 años.

Debe señalarse que el cálculo probabilístico del peligro sísmico se basa en el conjunto de modelos de zonas sísmicas y leyes de atenuación adoptados, y los análisis de confiabilidad son llevados a cabo con modelos estructurales y conjuntos de demandas de ductilidad simplificados. No se presenta ningún intento para desarrollar e incorporar modelos de peligro sísmico que pudieran igualar o mejorar los resultados del modelo empleado, ni tampoco para desarrollar e incorporar estructuras complejas (e.g., puentes) para la determinación del peligro sísmico y la evaluación de la confiabilidad. Aunque los sismos inslab e interplaca son los que más contribuyen al peligro sísmico para muchos sitios, para complementar el presente trabajo, la evaluación de la respuesta inelástica y el análisis de la confiabilidad necesita extenderse para considerar otros tipos de sismos y zonas sísmicas que pudieran afectar a México. Por lo tanto, una revisión cuidadosa de los resultados obtenidos, del modelo de peligro sísmico adoptado, así como también el posible consenso entre profesionales de la práctica y los desarrolladores de reglamentos, son necesarios para posibles implementaciones en el código de diseño.

\section{AGRADECIMIENTOS}

Se reconoce de manera especial el apoyo financiero del Consejo Nacional de Ciencia y Tecnología (CONACYT) de México, del Consejo de Investigación para las Ciencias Naturales e Ingeniería de Canadá (NSERC por sus iniciales en inglés), y del Instituto de Ingeniería de la UNAM. Agradecemos a K. Goda, Daniel García, Carlos Reyes, y Miguel Jaimes por la información proporcionada, y a J. A. Escobar, Orlando Díaz, y dos revisores anónimos por sus cuantiosos comentarios constructivos, sugerencias, y críticas.

\section{REFERENCIAS}

Adams, J, y S Halchuk (2003), "Fourth generation seismic hazard maps of Canada: values for over 650 Canadian localities intended for the 2005 National Building Code of Canada", Open-File 4459, Geological Survey of Canada, Ottawa, Ontario, Canada.

AASHTO (2007), LRFD bridge design specifications, 4th edition, American Association of State Transportation Officials, Washington D. C.

Atkinson, G M y D M Boore, (2003), "Empirical ground-motion relations for subduction-zone earthquakes and their application to cascadia and other regions", Bulletin of the Seismic Society of America, Vol. 93, pp. 1703-1729.

Bojórquez, E, S E Ruiz y A Terán-Gilmore (2008), "Reliability-based evaluation of steel structures using energy concepts”, Engineering Structures, Vol. 30, No. 6, pp. 1745-1759.

Boore, D M, W B Joyner and T E Fumal (1997), "Equations for estimating horizontal response spectra and peak acceleration from western North America", Seismic Research Letters, No. 68, pp. 128153. (for Erratum: see 2005, Seism Res. Lett., No. 76, pp. 368-369). 
Boore, D M (2005), "On pads and filters: processing strong-ground motion data", Bulletin of the Seismological Society of America, Vol. 95, No. 2, pp. 745-750.

CAN/CSA-S6 (2006), Canadian Standards Association, Canadian Highway Bridge Design Code, Toronto.

Cornell, C A (1968), "Engineering seismic risk analysis", Bulletin of the Seismic Society of America, Vol. 58, pp. 1583-1606.

Díaz-López, O, y L Esteva (1991), "Calibrating simplified models for computation of seismic reliability of multistory frames", in Proceeding of ICASP6 (eds. Esteva, L, and S Ruiz.), Institute of Engineering, Mexico, D.F., Mexico, pp. 328-337.

Ellingwood, B R (2001), "Earthquake risk assessment of building structures", Reliab. Engng. Syst. Safety, Vol. 74, pp. 251-262.

Ellingwood, B R, T V Galambos, J G MacGregor y C A Cornell (1980), "Development of a probability based load criterion for American National Standard A58", Spec. Pub. No. 577, National Bureau of Standards, Washington, D. C.

Esteva, L (1968), "Bases para la formulación de decisiones de diseño sísmico", Tesis doctoral, Universidad Nacional Autónoma de México, Ciudad de México, México.

Esteva, L, O Díaz-López, J García-Pérez, G Sierra y E Ismael (2002), "Life-cycle optimization in the establishment of performance-acceptance parameters for seismic design", Structural Safety, Vol. 24, pp. 187-204.

Frankel, A (1995), "Mapping seismic hazard in the central and eastern United States", Seismic Research Letters., Vol. 66, pp. 8-21.

Frankel, A, C Mueller, T Barnhard, D Perkins, E V Leyendecker, N Dickman, S Hanson, y M Hopper (1996), "National seismic hazard maps", Open-File 96-532, U.S. Department of the Interior, U.S. Geological Survey, Denver, CO.

García D, (2006), "Estimación de parámetros del movimiento fuerte del suelo para terremotos interplaca e intraslab en México Central", Tesis doctoral, Universidad Complutense de Madrid, Madrid, España.

García, D, S K Singh, M Herraiz, M Ordaz y J P Pacheco (2005), "Inslab earthquakes of Central Mexico: Peak ground-motion parameters and response spectra", Bulletin of the Seismic Society of America, Vol. 95, pp. 2272-2282.

García-Soto, A D, H P Hong y R Gómez (2012), "Effect of the orientation of records on displacement ductility demand", Canadian Journal of Civil Engineering, 39(4), 362-373.

Goda, K, y H P Hong (2006), "Optimum seismic design considering risk attitude, societal tolerable risk level and life quality criterion", Journal of Structural Engineering, ASCE, Vol. 132, No. 12, pp. 2027-2035.

Hong, H P, K Goda y A G Davenport (2006), "Seismic hazard analysis: a comparative study", Canadian Journal of Civil Engineering, Vol. 33 No. 9, pp. 1156-1171.

Hong, H P, y Hong P. (2007), "Assessment of ductility demand and reliability of bilinear single-degreeof-freedom-systems under earthquake loading”, Canadian Journal of Civil Engineering, 34(12), 1606-1615.

Hong, H P, A D García-Soto, y R Gomez (2010), "Impact of different earthquake types on the statistics of ductility demand”, Journal of Structural Engineering, ASCE, Vol. 136 No. 7, pp. 770-780. 
Hong, H P, A Pozos-Estrada y R Gomez (2009), "Orientation effect on ground motion measure for Mexican subduction earthquakes", Earthquake Engineering and Engineering Vibration, Vol. 8, No. 1, pp. 1-16.

Jaimes M A y E Reinoso (2006), "Comparación del comportamiento de edificios en el valle de México ante sismos de subducción y de falla normal”, Revista de Ingeniería Sísmica, No. 75, pp. 1-22.

Joyner, W B y D M Boore (1993), "Methods for regression analysis of strong-motion data", Bulletin of the Seismic Society of America, Vol. 83, pp. 469-487.

Madsen, H O, S Krenk, y N C Lind (1986), Methods of Structural Safety, Prentice-Hall, Inc., Englewood Cliffs, New Jersey.

McGuire, R K (1976), "FORTRAN Computer program for seismic risk analysis", Open-File 76-67, U.S. Department of the Interior, U.S. Geological Survey, Denver, CO.

McGuire, R K (2004), "Seismic hazard and risk analysis", Oakland, Calif.: Earthquake Engineering Research Institute.

MOC (1993), Manual de diseño de obras civiles, Diseño por sismo, Instituto de Investigaciones Eléctricas, Comisión Federal de Electricidad.

MOC (2008), Manual de diseño de obras civiles, Diseño por sismo. Recomendaciones y Comentarios, Instituto de Investigaciones Eléctricas, Comisión Federal de Electricidad.

Nakashima M (1997), Uncertainties associated with ductility performance of steel building structures, Seismic design methodology for the next generation of codes (eds. Fajfar P, Krawinkler H.), Balkema, Rotterdam.

NBCC (2005), National Building Code of Canada, Canadian Commission for Building and Fire Codes, National Research Council Canada, Ottawa.

NTCS (2004), Normas técnicas complementarias para diseño por sismo, Reglamento de Construcciones para el Distrito Federal, Gaceta Oficial del Departamento del Distrito Federal.

Ordaz, M y C Reyes (1999), "Earthquake hazard in Mexico City: Observations versus computations", Bulletin of the Seismic Society of America, Vol. 89, No. 5, pp. 1379-1383.

Ordaz, M, L E Pérez-Rocha y J Alemán Velásquez (2007), "Definición de sismos máximos para el diseño sísmico de estructuras", XVI Congreso Nacional de Ingeniería Sísmica, Ixtapa-Zihuatanejo, Guerrero, México.

Pardo, M y G Suárez (1995), "Shape of the subducted Rivera and Cocos plates in southern Mexico: Seismic and tectonic implications", Journal of Geophysical Research, Vol. 100, No. B7, pp. $12,357-12373$.

Pozos-Estrada, A, R Gómez y H P Hong (2008), "Seismic design: benefit/cost for overall service time versus per unit service time", Proceedings of the 14th World Conference on Earthquake Engineering, Beijing.

Rackwitz, R (2000), “Optimization-the basis of code-making and reliability verification", Structural Safety, Vol. 22, pp. 27-60.

Reyes, C, E Miranda, M Ordaz y R Meli (2002), "Estimación de espectros de aceleraciones correspondientes a diferentes periodos de retorno para las distintas zonas sísmicas de la ciudad de México", Revista de Ingeniería Sísmica, No. 66, pp. 95-121. 
Rosenblueth, E y L Esteva (1972), "Reliability Basis for Some Mexican Codes Publication", ACI Special Publication, Vol. 31, pp. 1-42.

Rosenblueth, E y R Gómez (1991), "Comentarios y ejemplos a las normas técnicas complementarias para diseño por sismo", DDF, Series de Instituto de Ingeniería, ES-7, UNAM, México.

Rosenblueth, E y J M Jara (1991), "Constant versus time dependent seismic design coefficients", Proceedings of the 3rd IFIP WG 7.5 Conference on Reliability and Optimization of Structural Systems '90, Springer-Verlag, Berlin, Germany, Vol. 1, pp. 315-327.

SCT (2001), Nueva normatividad para diseño de puentes, página electrónica del IMT y la SCT, http://normas.imt.mx/default.htm, N-PRY-CAR-6-01-001/01 a N-PRY-CAR-6-01-006/01, Secretaria de Comunicaciones y transportes.

Singh, S K, R Quaas, M Ordaz, F Mooser, D Almora, M Torres y R Vásquez (1995), "Is there truly a hard-rock site in the valley of Mexico", Geophysical Research Letters, Vol. 22, No. 4, pp. 481-484.

Tena-Colunga, A, U Mena, L E Pérez-Rocha, M Ordaz, J Avilés y J I Vilar (2008), "Updated guidelines for the seismic design of buildings in the MOC-2008 code of Mexico", Proceedings of the 14th World Conference on Earthquake Engineering, Beijing, China.

Zúñiga, R, G Suárez, M Ordaz y V García-Acosta (1997), "Peligro sísmico en Latinoamérica y el Caribe", Reporte Final IPGH. 